\title{
ON A NONLOCAL REACTION-DIFFUSION PROBLEM ARISING FROM THE MODELING OF PHYTOPLANKTON GROWTH*
}

\author{
YIHONG DU ${ }^{\dagger}$ AND SZE-BI HSU $\ddagger$
}

\begin{abstract}
In this paper we analyze a nonlocal reaction-diffusion model which arises from the modeling of competition of phytoplankton species with incomplete mixing in a water column. The nonlocal nonlinearity in the model describes the light limitation for the growth of the phytoplankton species. We first consider the single-species case and obtain a complete description of the longtime dynamical behavior of the model. Then we study the two-species competition model and obtain sufficient conditions for the existence of positive steady states and uniform persistence of the dynamical system. Our approach is based on a new modified comparison principle, fixed point index theory, global bifurcation arguments, elliptic and parabolic estimates, and various analytical techniques.
\end{abstract}

Key words. phytoplankton, competition for light, uniform persistence, reaction-diffusion equation, steady state

AMS subject classifications. 35J55, 35J65, 92D25

DOI. $10.1137 / 090775105$

1. Introduction. In this paper we analyze a reaction-diffusion model which describes the growth of phytoplankton species in a eutrophic environment. In such environments there are ample nutrients and the phytoplankton species typically compete for light. In $[17,18,25]$ Huisman and Weissing developed a theory of interspecific competition for light that assumes complete mixing of phytoplankton species. This theory is based on a system of ordinary differential equations (ODEs) and predicts that complete mixing leads to competitive exclusion similar to that in $[13,12,1,23]$; namely, the species with the lowest "critical light intensity" wins the competition.

However, in many aquatic environments, phytoplankton species are not thoroughly mixed. To understand the effect of incomplete mixing on the growth of phytoplankton species in a eutrophic environment, Huisman, van Oostveen, and Weissing [16] introduced a reaction-diffusion model and analyzed the model through numerical simulations. But a thorough mathematical treatment of the model has been lacking. The purpose of this paper is to prove some basic mathematical facts for this model which provide a basis for further rigorous mathematical analysis of the involved reaction-diffusion system. Our uniform persistence result indicates that with incomplete mixing of the phytoplankton species, competitive exclusion does not always happen, and coexistence can occur in some parameter ranges.

* Received by the editors October 27, 2009; accepted for publication (in revised form) February 23, 2010; published electronically May 28, 2010.

http://www.siam.org/journals/sima/42-3/77510.html

†School of Science and Technology, University of New England, Armidale, NSW 2351, Australia, and Department of Mathematics, Qufu Normal University, Shandong, People's Republic of China (ydu@turing.une.edu.au). This author's research was partially supported by the Australian Research Council and NSFC 10571022. Part of this work was done during this author's visit to the National Center of Theoretical Sciences of Taiwan.

${ }^{\ddagger}$ Department of Mathematics and National Center of Theoretical Science, National Tsing-Hua University, Hsinchu, Taiwan 300, Republic of China (sbhsu@math.nthu.edu.tw). This author's research was partially supported by National Council of Science, Taiwan, Republic of China. Part of this work was done during this author's visit to the University of New England, Australia.

1305 
The model of [16] is one among many mathematical models of phytoplankton proposed and investigated in recent years; see $[20,9,14,10,15,21,26]$ and the references therein for related study on the formation of phytoplankton blooms from mathematical, experimental, and numerical viewpoints. It is our hope that some of the mathematical theory and techniques developed here for treating the model of [16] can also find applications in the study of other phytoplankton models. Some mathematical research closely related to the subject of this paper is mentioned at the end of this section in more detail.

We now briefly describe the model of [16]. Consider a water column with a cross section of one unit area and with $n$ phytoplankton species. Let $x$ denote the depth within the water column where $x$ runs from 0 (top) to $L$ (bottom). And let $u_{i}(x, t)$ denote the population density (numbers per unit volume) of a phytoplankton species $i$ at depth $x$ and time $t$. The rate of change in phytoplankton densities is described by the following system of reaction-diffusion equations:

$$
\left(u_{i}\right)_{t}=D_{i}\left(u_{i}\right)_{x x}+\left(g_{i}(I(x, t))-d_{i}\right) u_{i}, i=1,2, \ldots, n,
$$

where $g_{i}(I(x, t))$ is the specific growth rate of phytoplankton species $i$ as a function of light intensity $I(x, t), D_{i}$ is the diffusion coefficient, and $d_{i}$ is the loss rate of the phytoplankton species $i$. Assume that the water column is closed, with no phytoplankton species entering or leaving the column at the top or the bottom. Thus the following boundary conditions are satisfied:

$$
\left(u_{i}\right)_{x}(0, t)=\left(u_{i}\right)_{x}(L, t)=0, i=1,2, \ldots, n .
$$

The initial conditions are

$$
u_{i}(x, 0)=u_{i}^{0}(x) \geq 0,0 \leq x \leq L, i=1,2, \ldots, n .
$$

The specific growth rate $g_{i}(I)$ satisfies

$$
g_{i}(0)=0, g_{i}^{\prime}(I)>0 \text { for } I \geq 0 .
$$

A typical example of $g_{i}(I)$ takes the Michaelis-Menten form, $g_{i}(I)=m_{i} I /\left(a_{i}+I\right)$, where $m_{i}$ is the maximal growth rate and $a_{i}$ is the half saturation constant. The light intensity $I(x, t)$ takes the form

$$
I(x, t)=I_{0} e^{-k_{0} x} \exp \left(-\int_{0}^{x}\left[k_{1} u_{1}(s, t)+\cdots+k_{n} u_{n}(s, t)\right] d s\right),
$$

where $I_{0}$ is the incident light intensity, $k_{0}$ is the background turbidity that summarizes light absorption by all nonphytoplankton components, and $k_{i}$ is the specific light attenuation coefficient of the phytoplankton species $i$.

In this paper we consider only the single-species case $(n=1)$ and the two-species case $(n=2)$. For a single species we obtain a complete understanding of the dynamical behavior of the reaction-diffusion problem. We show the existence of a critical loss rate $d_{*}>0$, determined by an eigenvalue problem, such that when the loss rate $d$ lies in $\left(0, d_{*}\right)$, the population density $u(x, t)$ of the species stabilizes at a unique positive steady state as time $t$ goes to infinity, and $u(x, t)$ goes to 0 as $t \rightarrow \infty$ when $d \geq d_{*}$. Moreover, we obtain qualitative properties of the unique positive steady-state solution, which are crucial for the study of the multispecies model. For the two-species model, our results are partial; we obtain some existence results for positive steady states and prove uniform persistence of the system under suitable conditions. 
A main technical difficulty in our analysis is the lack of an "order-preserving property" of the single-species equation, caused by the nonlocal nature of the nonlinearity. Many key techniques for handling similar problems collapse for the model here because of this. In section 2, we study the steady state for the single-species equation based on a bifurcation approach (for existence) and various subtle analytical techniques (for uniqueness and other properties of the solution). Section 3 is devoted to the global dynamical behavior of the single-species equation, which relies on a comparison lemma and a boundedness lemma, and a key observation used in our proof is that the function $v(x, t):=\int_{0}^{x} u(y, t) d y$ satisfies an equation which has the order-preserving property (see (3.4)). In section 4, we consider the two-species model and prove the existence of positive steady states by making use of the fixed point index theory and global bifurcation arguments. In section 5, we prove the uniform persistence and some extinction results for the two species dynamical system under certain suitable conditions. The analysis in sections 4 and 5 relies heavily on our results for the single-species case in sections 2 and 3.

We end the introduction by mentioning some closely related mathematical research. In [19], a reaction-diffusion model for a single phytoplankton species was studied, and global dynamical behavior of the equation was determined, where the water column was assumed to have infinite depth, and the sinking effect of the phytoplankton species is included. In [24], the two-species model of a nature similar to [19] was considered, but only for the special case that the functions $g_{i}(I)(i=1,2)$ are linear. However, the proof of the main result in [24] seems to contain serious gaps (the proof of Lemma 7 in [24] does not seem complete; for example, under the assumption that two positive solutions exist, there are more possibilities than (i) and (ii) listed there). The single-species model in [19] but with finite water depth was considered in two recent papers [9] and [21]. In [9], for the special case that $g(I)=I^{\alpha}, \alpha \in(0,1]$, the authors showed that there is a critical water depth for the existence and uniqueness of positive steady-state solutions. This work covered the case of buoyant phytoplankton (apart from the sinking type as in [19]), and it also characterized the phytoplankton bloom (for both sinking and buoyant type) by some critical values of the vertical turbulent diffusion coefficient. Moreover, it investigated the phase transition curve by reducing the equation to a Bessel equation (by taking advantage of the special nonlinearity $\left.g(I)=I^{\alpha}\right)$. However, the stability of the steady state solution or the dynamical behavior of the parabolic equation was not considered. In [21], under suitable conditions, the existence and uniqueness of a positive steady state was proved, and it was also shown that the steady state is locally asymptotically stable. Our Theorem 3.3 below shows that the unique positive steady state is not only locally asymptotically stable, but it is also globally attractive. On the other hand, our Theorem 2.1 implies that the conditions imposed in [21] for the existence of a positive steady state are not sharp. (To be accurate, our results here cover only the special case that the sinking velocity $v$ is 0 in [21], but a simple modification of our techniques shows that both our Theorems 2.1 and 3.3 are valid for nonzero $v$.) In $[7,8]$, a reaction-diffusion model proposed by Klausmeier and Litchman [20] was examined, where both nutrient and light limitations for the growth of a single phytoplankton species were included, and the focus was on the location of biomass concentration under the assumption that, apart from passive diffusion caused by currents movement, the species actively move to the optimal spatial location for its growth (determined by the light and nutrient distributions). 
2. The steady states of a single population species. In this section we study the steady states of a single population growth, i.e., (1.1)-(1.5) with $n=1$, namely,

$$
\begin{aligned}
& u_{t}=D u_{x x}+(g(I(x, t))-d) u, 0<x<L, t>0, \\
& u_{x}(0, t)=0, u_{x}(L, t)=0, t>0, \\
& u(x, 0)=u_{0}(x) \supsetneqq 0,0 \leq x \leq L,
\end{aligned}
$$

where $g \in C^{1}([0, \infty))$ satisfies

$$
\begin{aligned}
& g(0)=0 \text { and } g \text { is strictly increasing, } \\
& I(x, t)=I_{0} e^{-k_{0} x} \exp \left(-k \int_{0}^{x} u(s, t) d s\right), I_{0}, k_{0}, k>0 .
\end{aligned}
$$

With suitable scaling, we may assume that $L=1$, and by replacing $g(\cdot)$ by $g\left(I_{0} \cdot\right)$ we may assume $I_{0}=1$. With these conventions the steady-state problem becomes

$$
-D u^{\prime \prime}=\left[g\left(e^{-k_{0} x} e^{-k \int_{0}^{x} u(s) d s}\right)-d\right] u \text { in }(0,1), u^{\prime}(0)=u^{\prime}(1)=0,
$$

where $D, k_{0}, k$, and $d$ are positive constants, and $g:[0, \infty) \rightarrow[0, \infty)$ is a $C^{1}$ increasing function, with $g(0)=0$. follow:

The following eigenvalue problem will play an important role in our analysis to

$$
\left\{\begin{array}{c}
-D \phi^{\prime \prime}+\Psi(x) \phi=\lambda \phi \text { in }(0,1), \\
\phi^{\prime}(0)=0, \phi^{\prime}(1)=0,
\end{array}\right.
$$

where $\Psi(x)$ is a continuous function in $[0,1]$. It is well known that $(2.7)$ has a smallest eigenvalue $\lambda_{1}=\lambda_{1}(\Psi)$, which corresponds to a positive eigenfunction $\phi_{1}$, and $\lambda_{1}$ is the only eigenvalue whose corresponding eigenfunction does not change sign. Moreover, $\Psi_{1} \geq \Psi_{2}$ implies $\lambda_{1}\left(\Psi_{1}\right) \geq \lambda_{1}\left(\Psi_{2}\right)$, and equality holds only if $\Psi_{1} \equiv \Psi_{2} ; \Psi_{n} \rightarrow \Psi$ in $C([0,1])$ implies $\lambda_{1}\left(\Psi_{n}\right) \rightarrow \lambda_{1}(\Psi)$.

Define

$$
\Psi_{0}(x):=-g\left(e^{-k_{0} x}\right), \quad d_{*}:=-\lambda_{1}\left(\Psi_{0}\right) .
$$

We are now ready to state and prove the main result of this section.

Theorem 2.1. Problem (2.6) has a unique positive solution for $d \in\left(0, d_{*}\right)$, and it has no positive solution if $d \notin\left(0, d_{*}\right)$. Moreover, if we denote the unique positive solution by $u_{d}$, then

(i) $d \rightarrow u_{d}$ is continuous from $\left(0, d_{*}\right)$ to $C^{2}([0,1])$,

(ii) $0<d_{1}<d_{2}<d_{*}$ implies $u_{d_{1}}(0)>u_{d_{2}}(0)$,

(iii) $u_{d} \rightarrow \infty$ uniformly on $[0,1]$ as $d \rightarrow 0$,

(iv) $0<d_{1}<d_{2}<d_{*}$ implies $\int_{0}^{x} u_{d_{1}}(s) d s>\int_{0}^{x} u_{d_{2}}(s) d s$ for all $x \in(0,1]$.

Proof. It follows from a standard bifurcation argument of Crandall and Rabinowitz [3] and Rabinowitz [22] that (2.6) has an unbounded branch of positive solutions, which we denote as $\Gamma=\{(d, u)\} \subset R^{1} \times C^{1}([0,1])$, that bifurcates from the trivial solution branch $\{(d, 0)\}$ at $\left(d_{*}, 0\right)$. If $(d, u)$ is a positive solution of $(2.6)$, then from the equation we deduce

$$
-d=\lambda_{1}\left[-g\left(e^{-k_{0} x} e^{-k \int_{0}^{x} u(s) d s}\right)\right] \in\left(\lambda_{1}\left[-g\left(e^{-k_{0} x}\right)\right], \lambda_{1}(0)\right)=\left(-d_{*}, 0\right) .
$$


That is,

$$
0<d<d_{*} .
$$

Therefore (2.6) has no positive solution when $d \notin\left(0, d_{*}\right)$.

We show next that the branch $\Gamma$ can only become unbounded through $(d, u) \in \Gamma$ satisfying $d \rightarrow 0$ and $\|u\|_{\infty} \rightarrow \infty$. We argue indirectly and assume that there exists $\left(d_{n}, u_{n}\right) \in \Gamma$ satisfying $d_{n} \rightarrow d_{0} \in\left(0, d_{*}\right]$ and $\left\|u_{n}\right\|_{\infty} \rightarrow \infty$. Denote $\hat{u}_{n}=u_{n} /\left\|u_{n}\right\|_{\infty}$. Then

$$
-D \hat{u}_{n}^{\prime \prime}=\left[g\left(e^{-k_{0} x} e^{-k \int_{0}^{x} u_{n}(s) d s}\right)-d_{n}\right] \hat{u}_{n} \text { in }(0,1), \hat{u}_{n}^{\prime}(0)=\hat{u}_{n}^{\prime}(1)=0 .
$$

Therefore $\left\{\hat{u}_{n}\right\}$ and $\left\{\hat{u}_{n}^{\prime \prime}\right\}$ are both bounded sequences in $L^{\infty}([0,1])$. By standard $L^{p}$ theory of elliptic equations, $\left\{\hat{u}_{n}\right\}$ is bounded in $W^{2, p}([0,1])$ for any $p>1$, and hence, by the Sobolev embedding theorem, it is precompact in $C^{1}([0,1])$. By passing to a subsequence, we may assume that $\hat{u}_{n} \rightarrow \hat{u}$ in $C^{1}([0,1])$. Since $f_{n}(x):=$ $g\left(e^{-k_{0} x} e^{-k \int_{0}^{x} u_{n}(s) d s}\right)$ is a bounded sequence in $L^{\infty}([0,1])$, by passing to a subsequence, we may assume that $f_{n} \rightarrow f$ weakly in $L^{2}([0,1])$. We note that $0 \leq f \leq g(1)$ in $[0,1]$ since each $f_{n}$ has this property. It is now easily seen that $\hat{u}$ is a weak solution of

$$
-D \hat{u}^{\prime \prime}=\left(f-d_{0}\right) \hat{u} \text { in }(0,1), \hat{u}^{\prime}(0)=\hat{u}^{\prime}(1)=0,
$$

and $\hat{u} \geq 0,\|\hat{u}\|_{\infty}=1$. Since $\left(f-d_{0}\right) \in L^{\infty}([0,1])$, we can apply the strong maximum principle to conclude that $\hat{u}>0$ on $[0,1]$ and $-d_{0}=\lambda_{1}(-f)$.

On the other hand, from $\hat{u}_{n} \rightarrow \hat{u}>0$ uniformly in $[0,1]$ and $\left\|u_{n}\right\|_{\infty} \rightarrow \infty$, we deduce that $u_{n} \rightarrow \infty$ uniformly on $[0,1]$. It follows that

$$
e^{-k \int_{0}^{x} u_{n}(s) d s} \rightarrow 0
$$

uniformly on any compact subset of $(0,1]$. This implies that $f \equiv 0$ and hence

$$
-d_{0}=\lambda_{1}(-f)=\lambda_{1}(0)=0
$$

a contradiction to our assumption that $d_{0} \in\left(0, d_{*}\right]$. Therefore $\Gamma$ can only become unbounded through the existence of a sequence $\left(d_{n}, u_{n}\right) \in \Gamma$ such that $d_{n} \rightarrow 0$ and $\left\|u_{n}\right\|_{\infty} \rightarrow \infty$; moreover, the above proof shows that in such a case, $u_{n} \rightarrow \infty$ uniformly on $[0,1]$. (In fact, $u_{n} /\left\|u_{n}\right\|_{\infty} \rightarrow 1$ in $C^{1}([0,1])$.)

As a consequence of the connectedness of $\Gamma$, we conclude that (2.6) has at least one positive solution for each $d \in\left(0, d_{*}\right)$.

We next prove the uniqueness conclusion. Suppose by way of contradiction that for some $d \in\left(0, d_{*}\right)$, (2.6) has two positive solutions, $u_{1}$ and $u_{2}$. We first observe that $u_{1}-u_{2}$ must change sign in $(0,1)$. Otherwise we may assume that $u_{1} \leq u_{2}$ and $u_{1} \not \equiv u_{2}$ in $[0,1]$. From this and the equations for $u_{1}$ and $u_{2}$ we deduce

$$
-d=\lambda_{1}\left[-g\left(e^{-k_{0} x} e^{-k \int_{0}^{x} u_{1}(s) d s}\right)\right]<\lambda_{1}\left[-g\left(e^{-k_{0} x} e^{-k \int_{0}^{x} u_{2}(s) d s}\right)\right]=-d,
$$

a contradiction. Therefore $u_{1}-u_{2}$ changes sign in $(0,1)$.

We claim that $u_{1}(0) \neq u_{2}(0)$. Otherwise, for $i=1,2$, we denote $v_{i}(x)=$ $\int_{0}^{x} u_{i}(s) d s, w_{i}(x)=u_{i}^{\prime}(x)$, and find that $\left(u_{i}, v_{i}, w_{i}\right)$ are solutions of the initial value system

$$
\left(u^{\prime}, v^{\prime}, w^{\prime}\right)=\left(w, u,-D^{-1}\left[g\left(e^{-k_{0} x} e^{-k v}\right)-d\right] u\right),(u(0), v(0), w(0))=\left(u_{1}(0), 0,0\right) .
$$


By the well-known existence and uniqueness theorem of ODEs, we conclude that $\left(u_{1}, v_{1}, w_{1}\right) \equiv\left(u_{2}, v_{2}, w_{2}\right)$ in a small neighborhood $[0, \delta)$. We may then repeat this argument to conclude that $u_{1} \equiv u_{2}$ as long as they are defined, which is a contradiction to our assumption that they are different solutions of (2.6). Therefore $u_{1}(0) \neq u_{2}(0)$.

For definiteness we assume that $u_{1}(0)<u_{2}(0)$. Since $u_{1}-u_{2}$ changes sign in $(0,1)$, there exists $x_{0} \in(0,1)$ such that $u_{2}(x)>u_{1}(x)$ in $\left[0, x_{0}\right)$ and $u_{1}\left(x_{0}\right)=u_{2}\left(x_{0}\right)$. We have

$$
\int_{0}^{x_{0}}\left[-u_{1}^{\prime \prime} u_{2}\right] d x=D^{-1} \int_{0}^{x_{0}}\left[g\left(e^{-k_{0} x} e^{-k \int_{0}^{x} u_{1}(s) d s}\right)-d\right] u_{1} u_{2} d x .
$$

Using integration by parts, we deduce

$-\left.u_{1}^{\prime} u_{2}\right|_{0} ^{x_{0}}+\int_{0}^{x_{0}} u_{1}^{\prime} u_{2}^{\prime} d x=D^{-1} \int_{0}^{x_{0}} g\left(e^{-k_{0} x} e^{-k \int_{0}^{x} u_{1}(s) d s}\right) u_{1} u_{2} d x-D^{-1} d \int_{0}^{x_{0}} u_{1} u_{2} d x$.

Similarly,

$$
\int_{0}^{x_{0}}\left[-u_{2}^{\prime \prime} u_{1}\right] d x=D^{-1} \int_{0}^{x_{0}}\left[g\left(e^{-k_{0} x} e^{-k \int_{0}^{x} u_{2}(s) d s}\right)-d\right] u_{1} u_{2} d x
$$

and

$-\left.u_{2}^{\prime} u_{1}\right|_{0} ^{x_{0}}+\int_{0}^{x_{0}} u_{1}^{\prime} u_{2}^{\prime} d x=D^{-1} \int_{0}^{x_{0}} g\left(e^{-k_{0} x} e^{-k \int_{0}^{x} u_{2}(s) d s}\right) u_{1} u_{2} d x-D^{-1} d \int_{0}^{x_{0}} u_{1} u_{2} d x$.

Therefore

$$
\left.\left[u_{1} u_{2}^{\prime}-u_{1}^{\prime} u_{2}\right]\right|_{0} ^{x_{0}}=D^{-1} \int_{0}^{x_{0}}\left[g\left(e^{-k_{0} x} e^{-k \int_{0}^{x} u_{1}(s) d s}\right)-g\left(e^{-k_{0} x} e^{-k \int_{0}^{x} u_{2}(s) d s}\right)\right] u_{1} u_{2} d x .
$$

Since $u_{1}^{\prime}(0)=u_{2}^{\prime}(0)=0$ by the boundary condition, and $u_{1}\left(x_{0}\right)=u_{2}\left(x_{0}\right)$, $u_{1}^{\prime}\left(x_{0}\right) \geq u_{2}^{\prime}\left(x_{0}\right)$, we have

$$
\left.\left[u_{1} u_{2}^{\prime}-u_{1}^{\prime} u_{2}\right]\right|_{0} ^{x_{0}}=u_{1}\left(x_{0}\right)\left[u_{2}^{\prime}\left(x_{0}\right)-u_{1}^{\prime}\left(x_{0}\right)\right] \leq 0 .
$$

Therefore (2.9) implies that

$$
\int_{0}^{x_{0}}\left[g\left(e^{-k_{0} x} e^{-k \int_{0}^{x} u_{1}(s) d s}\right)-g\left(e^{-k_{0} x} e^{-k \int_{0}^{x} u_{2}(s) d s}\right)\right] u_{1} u_{2} d x \leq 0 .
$$

But on the other hand, from $u_{1}(x)<u_{2}(x)$ in $\left(0, x_{0}\right)$ we deduce

$$
\int_{0}^{x_{0}}\left[g\left(e^{-k_{0} x} e^{-k \int_{0}^{x} u_{1}(s) d s}\right)-g\left(e^{-k_{0} x} e^{-k \int_{0}^{x} u_{2}(s) d s}\right)\right] u_{1} u_{2} d x>0 .
$$

This contradiction proves our uniqueness conclusion, and we can now denote the unique positive solution of (2.6) by $u_{d}$.

The fact that $d \rightarrow u_{d}$ is continuous as a map from $\left(0, d_{*}\right)$ to $C^{1}([0,1])$ follows from a standard compactness and uniqueness consideration: If $d_{n} \rightarrow d_{0} \in\left(0, d_{*}\right)$, then a subsequence of $u_{d_{n}}$ converges in $C^{1}([0,1])$ to a positive solution of $(2.6)$ with $d=d_{0}$. By uniqueness, this positive solution must be $u_{d_{0}}$. Therefore the entire sequence converges to $u_{d_{0}}$. Moreover, from the equation of $u_{d_{n}}$ we easily see that $u_{d_{n}} \rightarrow u_{d_{0}}$ in 
$C^{1}([0,1])$ implies that the convergence also holds in $C^{2}([0,1])$. Conclusion (i) is now proved.

We now show that $0<d_{1}<d_{2}<d_{*}$ implies $u_{d_{1}}(0)>u_{d_{2}}(0)$. To simplify the notation, we will write $u_{1}=u_{d_{1}}, u_{2}=u_{d_{2}}$ in the following discussion.

We argue indirectly and assume that for some $0<d_{1}<d_{2}<d_{*}$ the inequality $u_{1}(0) \leq u_{2}(0)$ holds. Consider first the case $u_{1}(0)<u_{2}(0)$. Then we can show $u_{1}-u_{2}$ changes sign and define $\left[0, x_{0}\right]$ as in the above uniqueness proof. We similarly have

$$
\left.\left[u_{1} u_{2}^{\prime}-u_{1}^{\prime} u_{2}\right]\right|_{0} ^{x_{0}} \leq 0
$$

On the other hand,

$$
\begin{aligned}
{\left.\left[u_{1} u_{2}^{\prime}-u_{1}^{\prime} u_{2}\right]\right|_{0} ^{x_{0}} } & =\int_{0}^{x_{0}}\left[-u_{1}^{\prime \prime} u_{2}+u_{2}^{\prime \prime} u_{1}\right] d x \\
& =D^{-1} \int_{0}^{x_{0}}\left[g\left(e^{-k_{0} x} e^{-k \int_{0}^{x} u_{1}(s) d s}\right)-g\left(e^{-k_{0} x} e^{-k \int_{0}^{x} u_{2}(s) d s}\right)\right] u_{1} u_{2} d x \\
& +D^{-1}\left(d_{2}-d_{1}\right) \int_{0}^{x_{0}} u_{1} u_{2} d x \\
& >0
\end{aligned}
$$

a contradiction.

Consider now the case $u_{1}(0)=u_{2}(0)$. Then from (2.6) and $d_{2}>d_{1}$ we find that $u_{2}^{\prime \prime}(0)>u_{1}^{\prime \prime}(0)$. Since $u_{1}^{\prime}(0)=u_{2}^{\prime}(0)=0$, it follows that $u_{2}(x)>u_{1}(x)$ for $x>0$ small. Thus we can still find an interval $\left(0, x_{0}\right)$ as above and derive a contradiction. Therefore $u_{1}(0)>u_{2}(0)$. Conclusion (ii) is now proved.

Conclusion (iii) follows from our argument earlier, where we proved that $\Gamma$ can become unbounded only through a sequence $\left(d_{n}, u_{n}\right) \in \Gamma$ with $d_{n} \rightarrow 0$ and $\left\|u_{n}\right\|_{\infty} \rightarrow \infty$.

We now consider (iv). We observe that if $u$ is a positive solution of (2.6), then, by integrating (2.6) from 0 to $x, v(x):=\int_{0}^{x} u(s) d s$ satisfies

$$
\begin{aligned}
-D v^{\prime \prime} & =-d v+\int_{0}^{x} g\left(e^{-k_{0} s-k v(s)}\right) u(s) d s \\
& =-d v+k^{-1} \int_{0}^{x} g\left(e^{-k_{0} s-k v(s)}\right) d\left(k_{0} s+k v(s)\right)-k_{0} k^{-1} \int_{0}^{x} g\left(e^{-k_{0} s-k v(s)}\right) d s \\
& =-d v+k^{-1} \int_{0}^{k_{0} x+k v(x)} g\left(e^{-\xi}\right) d \xi-k_{0} k^{-1} \int_{0}^{x} g\left(e^{-k_{0} s-k v(s)}\right) d s \\
& =-d v+G\left(k_{0} x+k v(x)\right)-k_{0} k^{-1} \int_{0}^{x} g\left(e^{-k_{0} s-k v(s)}\right) d s,
\end{aligned}
$$

where

$$
G(\eta):=k^{-1} \int_{0}^{\eta} g\left(e^{-\xi}\right) d \xi .
$$

(The use of the function $G$ in the above equation for $v$ was motivated by [19].)

For $d \in\left(0, d_{*}\right)$ we set $v_{d}(x)=\int_{0}^{x} u_{d}(s) d s$. Fixing any $d_{2} \in\left(0, d_{*}\right)$, we want to show that $v_{d_{1}}(x)>v_{d_{2}}(x)$ for all $x \in(0,1]$ if $0<d_{1}<d_{2}$. To simplify the notation, whenever no confusion is caused, we write $u_{i}=u_{d_{i}}$ and $v_{i}=v_{d_{i}}$. By (iii), if $d_{1}>0$ is small enough, we have $u_{1}>u_{2}$ on $[0,1]$ and hence $v_{1}>v_{2}$ for $x \in(0,1]$. If the desired conclusion does not hold, then we can find a maximal $d_{1}<d_{2}$ such that $v_{d}(x)>v_{d_{2}}(x)$

Copyright $@$ by SIAM. Unauthorized reproduction of this article is prohibited. 
in $(0,1]$ for $d \in\left(0, d_{1}\right)$. Then clearly $v_{d_{1}} \geq v_{d_{2}}$. We claim that $v_{d_{1}}(x)=v_{d_{2}}(x)$ holds for some $x \in(0,1]$. Otherwise $v_{1}(x)>v_{2}(x)$ for all $x \in(0,1]$. Fix $d_{0} \in\left(d_{1}, d_{2}\right)$. By (ii), for any $d \in\left[d_{1}, d_{0}\right], u_{d}(0) \geq u_{d_{0}}(0)>u_{d_{2}}(0)$. By (i), there exists $C>0$ such that $\left\|u_{d}\right\|_{C^{2}([0,1])}<C$ for all $d \in\left[d_{1}, d_{0}\right]$. Therefore we can find $\delta>0$ small enough such that $v_{d}(x)>v_{d_{2}}(x)$ for $d \in\left[d_{1}, d_{0}\right]$ and $x \in(0, \delta]$. Since $v_{1}(x)>v_{2}(x)$ in $[\delta, 1]$, by (i) we can find $\tilde{d}_{1} \in\left(d_{1}, d_{0}\right]$ such that $v_{d}(x)>v_{d_{2}}(x)$ for $d \in\left[d_{1}, \tilde{d}_{1}\right]$ and $x \in[\delta, 1]$. Thus $v_{d}(x)>v_{d_{2}}(x)$ for $d \in\left(0, \tilde{d}_{1}\right]$ and $x \in(0,1]$, contradicting the maximality of $d_{1}$. This proves our claim that $v_{d_{1}}(x)=v_{d_{2}}(x)$ holds for some $x \in(0,1]$. We show that this leads to a contradiction.

Consider first the possibility that $x=1$, i.e., $v_{1}(1)=v_{2}(1)$. Since $v_{1}^{\prime \prime}(1)=v_{2}^{\prime \prime}(1)=$ 0 , we deduce from the above equation for $v$ that, for $i=1,2$,

$$
d_{i} v_{i}(1)=G\left(k_{0}+k v_{i}(1)\right)-k_{0} k^{-1} \int_{0}^{1} g\left(e^{-k_{0} s-k v_{i}(s)}\right) d s .
$$

Denote $\sigma:=v_{1}(1)=v_{2}(1)$. We obtain from the above identity

$$
\left(d_{2}-d_{1}\right) \sigma=k_{0} k^{-1} \int_{0}^{1}\left[g\left(e^{-k_{0} s-k v_{1}(s)}\right)-g\left(e^{-k_{0} s-k v_{2}(s)}\right)\right] d s .
$$

Since $v_{1} \geq v_{2}$, the right side of the above identity is less than or equal to 0 , but the left side is positive, and we arrive at a contradiction. Hence we necessarily have $v_{1}(1)>v_{2}(1)$.

Consider next the remaining possibility, namely, $x \in(0,1)$. Denoting $w:=v_{1}-v_{2}$, we obtain

$$
\begin{aligned}
-D w^{\prime \prime} & =d_{2} v_{2}-d_{1} v_{1}+C(x) w-k_{0} k^{-1} \int_{0}^{x}\left[g\left(e^{-k_{0} s-k v_{1}(s)}\right)-g\left(e^{-k_{0} s-k v_{2}(s)}\right)\right] d s \\
& \geq\left[C(x)-d_{1}\right] w, \\
w(0) & =0, w(1)>0,
\end{aligned}
$$

where $C(x)=G^{\prime}\left(k_{0} x+k \theta(x)\right)$ for some $\theta(x) \in\left[v_{2}(x), v_{1}(x)\right]$. By the strong maximum principle we deduce $w>0$ in $(0,1]$, again reaching a contradiction.

The proof is now complete.

3. The dynamics of a single population species. In this section we study the asymptotic behavior of the solution of the partial differential equation (2.1)-(2.3) satisfying (2.4) and (2.5). As before, without loss of generality we assume $I_{0}=1$, $L=1$ in $(2.1)-(2.5)$. The initial boundary value problem now has the form

$$
\begin{aligned}
& u_{t}=D u_{x x}+\left[g\left(e^{-k_{0} x-k \int_{0}^{x} u(s, t) d s}\right)-d\right] u, 0<x<1, t>0, \\
& u_{x}(0, t)=0, u_{x}(1, t)=0, t>0, \\
& u(x, 0)=u_{0}(x) \supsetneqq 0,0 \leq x \leq 1 .
\end{aligned}
$$

We assume that $u_{0} \in C([0,1])$.

By standard argument, it is not difficult to prove the uniqueness and global existence of the solution $u(x, t)$ of (3.1)-(3.3). By the maximum principle, $u(x, t)>0$ for $t>0$ and $x \in[0,1]$. Our aim here is to understand the long-time behavior of $u(x, t)$. 
Recall that $d_{*}=-\lambda_{1}\left(\Psi_{0}\right)$ is defined in (2.8). If $d>d_{*}$, then using

$$
u_{t} \leq D u_{x x}-d u+g\left(e^{-k_{0} x}\right) u
$$

and the comparison principle we deduce $u(x, t) \leq C e^{-\left(d-d_{*}\right) t} \phi_{1}(x) \rightarrow 0$ as $t \rightarrow \infty$ uniformly in $x \in[0,1]$, where $\phi_{1}$ is a positive eigenfunction corresponding to $\lambda_{1}\left(\Psi_{0}\right)$ and $C$ is a positive constant such that $u_{0}(x) \leq C \phi_{1}(x)$ in $[0,1]$. Therefore

$$
\lim _{t \rightarrow \infty} u(x, t)=0 \text { uniformly for } x \in[0,1] \text { if } d>d_{*} .
$$

We consider next the case $d \in\left(0, d_{*}\right)$; the case $d=d_{*}$ will be discussed last. So we suppose now $0<d<d_{*}$. By Theorem 2.1, (3.1)-(3.2) has a unique positive steady state $u_{d}(x)$. We will show that $u(x, t) \rightarrow u_{d}(x)$ as $t \rightarrow \infty$ uniformly for $x$ in $[0,1]$. To prove this conclusion, we need two key results, namely, a comparison lemma and a boundedness lemma.

Set

$$
v(x, t)=\int_{0}^{x} u(s, t) d s .
$$

Then $v(x, t)$ satisfies $v(0, t) \equiv 0$ and

$$
\begin{aligned}
v_{t} & =D v_{x x}-d v+\int_{0}^{x} g\left(e^{-k_{0} s-k v(s, t)}\right) u(s, t) d s \\
& =D v_{x x}-d v+G\left(k_{0} x+k v(x, t)\right)-k_{0} k^{-1} \int_{0}^{x} g\left(e^{-k_{0} s-k v(s, t)}\right) d s,
\end{aligned}
$$

where $G(\eta)=k^{-1} \int_{0}^{\eta} g\left(e^{-\xi}\right) d \xi$.

In the following two lemmas, we do not require $d \in\left(0, d_{*}\right)$.

Lemma 3.1 (comparison lemma). Suppose $d \in(-\infty, \infty)$ and $u, \tilde{u} \in C^{2,1}([0,1] \times$ $(0, \infty))$ satisfy

$$
\begin{aligned}
& u_{t} \leq D u_{x x}+\left[g\left(e^{-k_{0} x-k \int_{0}^{x} u(s, t) d s}\right)-d\right] u, 0 \leq x \leq 1, t>0, \\
& u_{x}(0, t)=0, u_{x}(1, t)=0, t>0,
\end{aligned}
$$

and

$$
\begin{aligned}
& \tilde{u}_{t} \geq D \tilde{u}_{x x}+\left[g\left(e^{-k_{0} x-k \int_{0}^{x} \tilde{u}(s, t) d s}\right)-d\right] \tilde{u}, 0 \leq x \leq 1, t>0, \\
& \tilde{u}_{x}(0, t)=0, \tilde{u}_{x}(1, t)=0, t>0 .
\end{aligned}
$$

If $u(x, t)<\tilde{u}(x, t)$ for $x \in[0,1]$ and all small $t \geq 0($ say $t \in[0, \epsilon])$, then $v(x, t)<\tilde{v}(x, t)$ for all $t>0$ and $x \in(0,1]$, where

$$
v(x, t)=\int_{0}^{x} u(s, t) d s, \quad \tilde{v}(x, t)=\int_{0}^{x} \tilde{u}(s, t) d s .
$$

Proof. Since $u(x, t)<\tilde{u}(x, t)$ for $t \geq 0$ small and $x \in[0,1]$ we have

$$
v(x, t)<\tilde{v}(x, t) \text { for } t \geq 0 \text { small and } x \in(0,1] .
$$

Suppose the conclusion of Lemma 3.1 is not true. Then there exists a finite maximal time denoted by $t^{*}$ such that (3.5) holds for every $t \in\left[0, t^{*}\right)$. Clearly $v\left(x, t^{*}\right) \leq \tilde{v}\left(x, t^{*}\right)$ for all $x \in[0,1]$. We claim that

$$
v\left(x, t^{*}\right)=\tilde{v}\left(x, t^{*}\right) \text { for some } x \in(0,1] .
$$

Copyright $@$ by SIAM. Unauthorized reproduction of this article is prohibited. 
Otherwise we have $v\left(x, t^{*}\right)<\tilde{v}\left(x, t^{*}\right)$ for all $x \in(0,1]$. Denote

$$
w(x, t)=\tilde{v}(x, t)-v(x, t) .
$$

Then $w(x, t) \geq 0$ for $0 \leq t \leq t^{*}, 0 \leq x \leq 1$, and

$$
\begin{aligned}
w_{t} \geq & D w_{x x}-d w+C(x, t) w+k_{0} k^{-1} \int_{0}^{x}\left[g\left(e^{-k_{0} s-k v(s, t)}\right)-g\left(e^{-k_{0} s-k \tilde{v}(s, t)}\right)\right] d s \\
\geq & D w_{x x}+[C(x, t)-d] w \text { for } 0 \leq x \leq 1, t \in\left(0, t^{*}\right] \\
& w(0, t)=0, w(1, t)>0 \text { for } 0<t \leq t^{*} \\
& w(x, 0)>0 \text { for } 0<x \leq 1
\end{aligned}
$$

where

$$
C(x, t)=G^{\prime}\left(k_{0} x+k \theta(x, t)\right), \quad \theta(x, t) \in[v(x, t), \tilde{v}(x, t)] .
$$

Thus we may use the strong maximum principle and the Hopf boundary lemma to conclude that $w(x, t)>0$ for $t \in\left(0, t^{*}\right]$ and $x \in(0,1]$, and $w_{x}\left(0, t^{*}\right)>0$. Then by the smoothness of $w(x, t)$, we obtain $w_{x}(x, t)>0$ for all $t$ close to $t^{*}$ and $x$ close to 0 . Thus from $w(0, t) \equiv 0$ we deduce $w(x, t)>0$ for $0<x \leq \delta, t^{*} \leq t \leq t^{*}+\delta, \delta>0$ small. From $w\left(x, t^{*}\right)>0$ for $x \in[\delta, 1]$, we can find $\delta_{0} \in(0, \delta)$ such that $w(x, t)>0$ for $x \in[\delta, 1]$ and $t \in\left[t^{*}, t^{*}+\delta_{0}\right]$. Thus $w(x, t)>0$ for $x \in(0,1]$ and $t \in\left(0, t^{*}+\delta_{0}\right]$, contradicting the maximality of $t^{*}$. This proves (3.6).

Thus there exists $x_{0} \in(0,1]$ such that $w\left(x_{0}, t^{*}\right)=0$. If $x_{0}=1$, i.e., $w\left(1, t^{*}\right)=0$, then $w_{t}\left(1, t^{*}\right) \leq 0$. By the boundary condition $v_{x x}\left(1, t^{*}\right)=u_{x}\left(1, t^{*}\right)=0, \tilde{v}_{x x}\left(1, t^{*}\right)=$ $\tilde{u}_{x}\left(1, t^{*}\right)=0$, and hence $w_{x x}\left(1, t^{*}\right)=0$. Therefore we can use (3.7) to obtain

$$
0 \geq w_{t}\left(1, t^{*}\right) \geq k_{0} k^{-1} \int_{0}^{1}\left[g\left(e^{-k_{0} s-k v\left(s, t^{*}\right)}\right)-g\left(e^{-k_{0} s-k \tilde{v}\left(s, t^{*}\right)}\right)\right] d s .
$$

Since $v\left(x, t^{*}\right) \leq \tilde{v}\left(x, t^{*}\right)$ in $[0,1]$, the above inequality holds only if $v\left(x, t^{*}\right) \equiv \tilde{v}\left(x, t^{*}\right)$, which implies $u\left(x, t^{*}\right) \equiv \tilde{u}\left(x, t^{*}\right)$.

From the inequality in $(3.7), w(x, t)$ is an upper solution of the problem

$$
\left\{\begin{aligned}
\tilde{w}_{t} & =D \tilde{w}_{x x}-d \tilde{w}, & & 0<x<1,0<t \leq t^{*}, \\
\tilde{w}(0, t) & =\tilde{w}(1, t)=0, & & 0<t \leq t^{*}, \\
\tilde{w}(x, 0) & =w(x, 0)>0, & & 0<x<1 .
\end{aligned}\right.
$$

By the strong maximum principle, $\tilde{w}(x, t)>0$ for $x \in(0,1)$ and $0<t \leq t^{*}$. On the other hand, by the comparison principle, we have $w(x, t) \geq \tilde{w}(x, t)$ for $x \in(0,1)$ and $0<t \leq t^{*}$. Hence $w\left(x, t^{*}\right)>0$ for $x \in(0,1)$. This contradicts our earlier conclusion that $w\left(x, t^{*}\right) \equiv 0$. Therefore we must have $w\left(1, t^{*}\right)>0$. We may now apply the strong maximum principle to (3.7) to conclude that $w\left(x, t^{*}\right)>0$ for $x \in(0,1]$, which is a contradiction to (3.6). The proof is now complete.

Lemma 3.2 (boundedness lemma). Suppose $d>0$, and let $u(x, t)$ be the unique solution of (3.1)-(3.3). Then there exists $C>0$ such that

$$
u(x, t) \leq C \text { for all } x \in[0,1], t>0 .
$$

Proof. Our assumption on $g$ implies that

$$
g(I) \leq \sigma I \text { for some } \sigma>0 \text { and all } I \in[0,1] .
$$

Copyright $@$ by SIAM. Unauthorized reproduction of this article is prohibited. 
Therefore with

$$
I(x, t)=\exp \left(-k_{0} x-k \int_{0}^{x} u(y, t) d y\right)
$$

we have

$$
g(I(x, t)) \leq \sigma I(x, t) \leq \sigma e^{-k \int_{0}^{x} u d y},
$$

and from the equation for $u$ we deduce that

$$
u_{t} \leq D u_{x x}+\left[\sigma e^{-k \int_{0}^{x} u d y}-d\right] u
$$

Integrating for $x$ from 0 to 1 , we obtain

$$
\left[\int_{0}^{1} u d x\right]_{t} \leq \sigma \int_{0}^{1} e^{-k \int_{0}^{x} u d y} u d x-d \int_{0}^{1} u d x .
$$

Denote

$$
w(t)=\int_{0}^{1} u(x, t) d x, \quad v(x, t)=\int_{0}^{x} u(y, t) d y .
$$

Then

$$
\begin{aligned}
\int_{0}^{1} e^{-k \int_{0}^{x} u d y} u d x & =\int_{0}^{1} e^{-k v} v_{x} d x \\
& =k^{-1}\left[e^{-k v(0, t)}-e^{-k v(1, t)}\right] \\
& =k^{-1}\left[1-e^{-k w(t)}\right]
\end{aligned}
$$

Therefore,

$$
w_{t} \leq \sigma k^{-1}\left[1-e^{-k w}\right]-d w
$$

and

$$
w_{t}+d w \leq C_{0}:=\sigma k^{-1}
$$

It follows that

$$
\left(e^{d t} w\right)_{t} \leq C_{0} e^{d t}
$$

from which we deduce

$$
w(t) \leq w(0) e^{-d t}+C_{0} e^{-d t} \int_{0}^{t} e^{d s} d s \leq C:=w(0)+C_{0} / d .
$$

To show the boundedness of $u(x, t)$ we set

$$
W(t):=\max _{x \in[0,1], s \in[0, t]} u(x, s) .
$$

Clearly $W(t)$ is nondecreasing. Suppose for contradiction that $W(t) \rightarrow \infty$ as $t \rightarrow \infty$.

Copyright (c) by SIAM. Unauthorized reproduction of this article is prohibited. 
Since $W(t) \rightarrow \infty$, we can find $t_{n} \rightarrow \infty$ such that $W\left(t_{n}\right)=\max _{x \in[0,1]} u\left(x, t_{n}\right)$. We may assume that $t_{n}>1$ for all $n \geq 1$. We then define

$$
v_{n}(x, t)=\frac{u\left(x, t+t_{n}-1\right)}{W\left(t_{n}\right)} .
$$

Clearly $v_{n}$ satisfies

$$
\left\{\begin{aligned}
\left(v_{n}\right)_{t} & =D\left(v_{n}\right)_{x x}+c_{n} v_{n} \\
\left(v_{n}\right)_{x} & =0 \text { for } x \in\{0,1\} \text { and } t>0 \\
v_{n}(x, 0) & \in[0,1]
\end{aligned}\right.
$$

where $c_{n}(x, t)=g\left(I\left(x, t+t_{n}-1\right)\right)-d$, and $\left|c_{n}\right| \leq M_{0}:=\max _{I \in[0,1]}|g(I)-d|$. A simple comparison consideration gives

$$
0 \leq v_{n}(x, t) \leq e^{M_{0} t} \text { for } x \in[0,1] \text { and } t \geq 0 .
$$

Hence we may apply standard parabolic regularity to conclude that $\left\{v_{n}\right\}$ is bounded in $C^{1+\alpha, \alpha}\left([0,1] \times\left[\frac{1}{2}, 2\right]\right)$ for any $\alpha \in(0,1)$. Therefore by passing to a subsequence if necessary we have $v_{n} \rightarrow v^{*}$ in $C^{1,0}\left([0,1] \times\left[\frac{1}{2}, 2\right]\right)$. Since $\left|c_{n}\right| \leq M_{0}$, by passing to a further subsequence, we may assume that $c_{n} \rightarrow c$ weakly in $L^{2}\left([0,1] \times\left[\frac{1}{2}, 2\right]\right)$. Clearly we have $|c| \leq M_{0}$. It follows that $v^{*}$ is a weak solution to

$$
\begin{cases}v_{t}^{*}=D v_{x x}^{*}+c v^{*} & \text { for } x \in[0,1], t \in[1 / 2,2], \\ v_{x}^{*}=0 & \text { for } x \in\{0,1\} \text { and } t \in[1 / 2,2], \\ v^{*} \in\left[0, e^{2 M_{0}}\right] & \text { for } x \in[0,1], t \in[1 / 2,2] .\end{cases}
$$

Since $\max _{x \in[0,1]} v_{n}(x, 1)=1$, we have $\max _{x \in[0,1]} v^{*}(x, 1)=1$ and hence $v^{*}$ is not identically zero. By the strong maximum principle we deduce $v^{*}(x, 1) \geq \delta_{0}>0$ in $[0,1]$. It follows that $v_{n}(x, 1) \geq \delta_{0} / 2$ for all large $n$ and $x \in[0,1]$. Therefore

$$
u\left(x, t_{n}\right) \geq\left(\delta_{0} / 2\right) W\left(t_{n}\right) \text { for all large } n \text { and } x \in[0,1] .
$$

But then we deduce

$$
w\left(t_{n}\right) \geq\left(\delta_{0} / 2\right) W\left(t_{n}\right) \rightarrow \infty
$$

as $n \rightarrow \infty$, which contradicts (3.9). Therefore there exists $C>0$ such that

$$
u(x, t) \leq C \text { for all } x \in[0,1] \text { and } t>0 .
$$

The proof is complete.

We are now ready to prove the main result of this section.

Theorem 3.3. Let $0<d<d^{*}$. Then the solution $u(x, t)$ of $(3.1)-(3.3)$ converges to the unique steady state $u_{d}(x)$ as $t \rightarrow \infty$ uniformly in $x \in[0,1]$.

Proof. We may assume that the initial data $u_{0}$ satisfies $u_{0}>0$ in $[0,1]$, for otherwise we can replace $u(x, t)$ by $u(x, 1+t)$ and $u_{0}(x)$ by $u(x, 1)$.

Since $d<d^{*}=-\lambda_{1}\left(\Psi_{0}\right)$ and

$$
\Psi_{\delta}(x):=-g\left(e^{-\left(k_{0}+k \delta\right) x}\right) \rightarrow \Psi_{0}(x)
$$

uniformly in $[0,1]$ as $\delta \rightarrow 0$, we can find $\delta>0$ sufficiently small such that $d<-\lambda_{1}\left(\Psi_{\delta}\right)$. Fix such a $\delta$ and let $\phi$ be a positive eigenfunction corresponding to $\lambda_{1}\left(\Psi_{\delta}\right)$. Then we 
choose $\epsilon>0$ small so that $\epsilon \phi<u_{0}, \epsilon \phi<\delta$ in $[0,1]$. Let $\underline{u}(x, t)$ be the unique solution of (3.1)-(3.2) with initial condition $\underline{u}(x, 0)=\epsilon \phi(x)$. Then we can find $\sigma>0$ small such that

$$
0<\underline{u}(x, t)<\delta \text { for } t \in(0, \sigma] \text { and } x \in[0,1] .
$$

Hence for $t \in(0, \sigma]$,

$$
\begin{aligned}
\underline{u}_{t} & =D \underline{u}_{x x}+\left[g\left(e^{-k_{0} x-k \int_{0}^{x} \underline{u}(y, t) d y}\right)-d\right] \underline{u} \\
& \geq D \underline{u}_{x x}+\left[-\Psi_{\delta}(x)-d\right] \underline{u} \\
& >D \underline{u}_{x x}+\left[-\Psi_{\delta}(x)+\lambda_{1}\left(\Psi_{\delta}\right)\right] \underline{u} .
\end{aligned}
$$

It follows that

$$
\begin{cases}(\underline{u}-\epsilon \phi)_{t}>D(\underline{u}-\epsilon \phi)_{x x}+\left[-\Psi_{\delta}(x)+\lambda_{1}\left(\Psi_{\delta}\right)\right](\underline{u}-\epsilon \phi), & x \in[0,1], t \in(0, \sigma], \\ (\underline{u}-\epsilon \phi)_{x}=0, & x=0,1, t \in(0, \sigma], \\ \underline{u}-\epsilon \phi=0, & x \in[0,1], t=0 .\end{cases}
$$

By the strong maximum principle we deduce $\underline{u}(x, t)-\epsilon \phi(x)>0$ for $t \in(0, \sigma]$ and $x \in[0,1]$. Fixing $s \in(0, \sigma]$, we thus have

$$
\underline{u}(x, s)>\underline{u}(x, 0) \text { in }[0,1] .
$$

By continuity,

$$
\underline{u}(x, s+t)>\underline{u}(x, t) \text { in }[0,1] \text { for all small } t \geq 0 .
$$

Thus we can use Lemma 3.1 to conclude that $\underline{v}(x, t)<\underline{v}(x, s+t)$ for $x \in[0,1]$ and $t>0$, where $\underline{v}(x, t)=\int_{0}^{x} \underline{u}(s, t) d s$. It follows that $\underline{v}(x, t)$ is monotone increasing in $t$.

By Lemma 3.2, $\underline{v}(x, t) \leq C$ for all $x \in[0,1]$ and $t>0$ for some $C>0$. Hence $\lim _{t \rightarrow \infty} \underline{v}(x, t)=v_{*}(x)$ exists. On the other hand, due to the boundedness of $\|\underline{u}(\cdot, t)\|_{\infty}$ (which follows from Lemma 3.2), we can apply the standard parabolic regularity theory to (3.1)-(3.2) to conclude that, for any sequence $t_{n} \rightarrow \infty,\left\{\underline{u}\left(\cdot, t_{n}\right)\right\}$ has a subsequence which converges in $C^{1}([0,1])$, say $\underline{u}\left(\cdot, t_{n_{k}}\right) \rightarrow u_{*}$. Since $\underline{v}\left(\cdot, t_{n}\right) \rightarrow v_{*}$, we necessarily have $v_{*}(x)=\int_{0}^{x} u_{*}(y) d y$. Hence $u_{*}=v_{*}^{\prime}$. This implies that $\lim _{t \rightarrow \infty} \underline{u}(x, t)$ exists and equals $v_{*}^{\prime}(x)$. It follows that $v_{*}^{\prime}$ must be a nonnegative steady state of (3.1), (3.2). Since $v_{*}(0)=0$ and $v_{*}$ is the limit of an increasing sequence, we have $v_{*}(x)>0$ for $x \in(0,1]$ and $v_{*}^{\prime} \not \equiv 0$. Therefore $v_{*}^{\prime}$ is a nontrivial nonnegative steady state of (3.1), (3.2). By the strong maximum principle $v_{*}^{\prime}$ is positive, and hence we can use Theorem 2.1 to conclude that $v_{*}^{\prime} \equiv u_{d}$.

Next we consider $d_{M}=-\lambda_{1}\left(\Psi_{M}\right)$ with $M>0$ large. Recall that $\Psi_{M}(x)=$ $-g\left(e^{-\left(k_{0}+k M\right) x}\right)$. Let $\phi_{M}(x)$ be the positive eigenfunction corresponding to $\lambda_{1}\left(\Psi_{M}\right)$ with $\left\|\phi_{M}\right\|_{\infty}=1$. It is easy to see by a regularity and compactness argument that as $M \rightarrow \infty, \lambda_{1}\left(\Psi_{M}\right) \rightarrow 0$ and $\phi_{M} \rightarrow 1$ in $C^{1}([0,1])$. Therefore we can find $M_{0}>0$ large so that

$$
d>-\lambda_{1}\left(\Psi_{M}\right), \frac{1}{2}<\phi_{M}(x) \leq 1 \text { for } M \geq M_{0} .
$$

We now fix $M>M_{0}$ such that $2 M>u_{0}(x)$ in $[0,1]$. Then

$$
u_{0}(x)<2 M \phi_{M}(x) \text { and } M<2 M \phi_{M}(x) \text { for } x \in[0,1] .
$$


Let $\bar{u}(x, t)$ be the solution of (3.1)-(3.2) with initial condition $\bar{u}(x, 0)=2 M \phi_{M}(x)$. Then we can find $\delta_{0}>0$ small so that $u_{0}(x)<\bar{u}(x, t), M<\bar{u}(x, t)$ for $t \in\left(0, \delta_{0}\right]$ and $x \in[0,1]$. Hence for $t \in\left(0, \delta_{0}\right]$, we have

$$
\begin{aligned}
\bar{u}_{t} & =D \bar{u}_{x x}+\left[g\left(e^{-k_{0} x-k \int_{0}^{x} \bar{u}(y, t) d y}\right)-d\right] \bar{u} \\
& \leq D \bar{u}_{x x}+\left[-\Psi_{M}(x)-d\right] \bar{u} \\
& <D \bar{u}_{x x}+\left[-\Psi_{M}(x)+\lambda_{1}\left(\Psi_{M}\right)\right] \bar{u} .
\end{aligned}
$$

Thus for $w(x, t):=\bar{u}(x, t)-2 M \phi_{M}(x)$, we have

$$
\begin{cases}w_{t}<D w_{x x}+\left[-\Psi_{M}(x)+\lambda_{1}\left(\Psi_{M}\right)\right] w, & x \in[0,1], t \in\left(0, \delta_{0}\right], \\ w_{x}=0, & x=0,1, t \in\left(0, \delta_{0}\right], \\ w=0, & x \in[0,1], t=0 .\end{cases}
$$

By the strong maximum principle we deduce $w=\bar{u}-2 M \phi_{M}<0$ for $t \in\left(0, \delta_{0}\right]$ and $x \in[0,1]$. It follows that $\bar{u}(x, s)<\bar{u}(x, 0)$ for $0<s \leq \delta_{0}$. Using the same argument as before, we deduce that

$$
\bar{v}(x, t):=\int_{0}^{x} \bar{u}(s, t) d s
$$

is monotone decreasing in $t$. Moreover, from Lemma 3.1 it follows that $\bar{v}(x, t)>$ $v(x, t):=\int_{0}^{x} u(s, t) d s>\underline{v}(x, t)$ for all $t>0$ and $x \in(0,1]$. Hence $\lim _{t \rightarrow \infty} \bar{v}(x, t)=$ $v^{*}(x) \geq \int_{0}^{x} u_{d}(s) d s$. We may then use parabolic regularity theory much as before to deduce that $\bar{u}(x, t) \rightarrow\left(v^{*}\right)^{\prime}(x)$ in $C^{1}([0,1])$, and $\left(v^{*}\right)^{\prime}(x)$ is a positive steady state of (3.1)-(3.2). Thus we must have $\left(v^{*}\right)^{\prime}(x) \equiv u_{d}(x)$. have

Since $\underline{v} \leq v \leq \bar{v}$ and $\lim _{t \rightarrow \infty} \underline{v}(x, t)=\lim _{t \rightarrow \infty} \bar{v}(x, t)=\int_{0}^{x} u_{d}(s) d s$, we necessarily

$$
\lim _{t \rightarrow \infty} v(x, t)=\int_{0}^{x} u_{d}(s) d s
$$

Thus we can repeat the above argument to conclude that $u(x, t) \rightarrow u_{d}(x)$ as $t \rightarrow \infty$ uniformly for $x \in[0,1]$. This completes the proof.

Finally we consider the case $d=d_{*}$.

Theorem 3.4. Suppose $d=d_{*}$. Then the solution $u(x, t)$ to (3.1)-(3.3) converges to 0 as $t \rightarrow \infty$ uniformly in $x \in[0,1]$.

Proof. This follows from a simple modification of the second part of the proof of Theorem 3.3. Indeed, let $\bar{u}(x, t)$ be defined exactly as in the proof of Theorem 3.3. Then we know that $\bar{v}(x, t):=\int_{0}^{x} \bar{u}(s, t) d s>0$ is strictly decreasing in $t$. Hence $\lim _{t \rightarrow \infty} \bar{v}(x, t)=v^{*}(x) \geq 0$ exists. By the same consideration as in the proof of Theorem 3.3 we can show that $\bar{u}(x, t) \rightarrow\left(v^{*}\right)^{\prime}(x)$ as $t \rightarrow \infty$ in the norm of $C^{1}([0,1])$, and hence $\left(v^{*}\right)^{\prime}(x)$ is a nonnegative steady state of (3.1)-(3.2). However, since $d=$ $d_{*}$, by Theorem 2.1, the only nonnegative steady state of (3.1)-(3.2) is the trivial solution 0 . Hence $\bar{u}(x, t) \rightarrow 0$ as $t \rightarrow \infty$ uniformly for $x \in[0,1]$, and thus $\bar{v}(x, t) \rightarrow 0$ as $t \rightarrow \infty$.

Using Lemma 3.1 we deduce $0<v(x, t)<\bar{v}(x, t)$, which implies that $v(x, t) \rightarrow 0$ as $t \rightarrow \infty$. Using this fact and the parabolic regularity, as before, we deduce $\lim _{t \rightarrow \infty} u(\cdot, t)$ exists in the $C^{1}([0,1])$ norm, and the limit is a nonnegative steady state of (3.1)-(3.2). Since $d=d_{*}$, this limit must be 0 . This completes the proof. 
4. Steady states of the two-species model. In this section we study the steady states of the system (1.1)-(1.3) with $n=2$. As before we assume, without loss of generality, that $L=1$ and $I_{0}=1$. Thus we are interested in the nonnegative solutions of the elliptic system

$$
\begin{cases}D_{1}\left(u_{1}\right)_{x x}+\left[g_{1}(I(x))-d_{1}\right] u_{1}=0, & 0<x<1, \\ D_{2}\left(u_{2}\right)_{x x}+\left[g_{2}(I(x))-d_{2}\right] u_{2}=0, & 0<x<1, \\ \left(u_{i}\right)_{x}(0)=\left(u_{i}\right)_{x}(1)=0, & i=1,2\end{cases}
$$

where $g_{1}(I), g_{2}(I)$ satisfy $(2.4)$, and

$$
I(x)=e^{-k_{0} x} \exp \left(-\int_{0}^{x}\left[k_{1} u_{1}(y)+k_{2} u_{2}(y)\right] d y\right),
$$

with $k_{0}, k_{1}, k_{2}>0$.

In the following we will regard $k_{0}, k_{1}, k_{2}$ as fixed constants and treat $d_{1}$ and $d_{2}$ as varying parameters. We need to introduce some notation first. We will use $\lambda_{1}(\Psi)$ to denote the first eigenvalue of $(2.7)$ with $D=D_{1}$ and use $\mu_{1}(\Psi)$ to denote the first eigenvalue of (2.7) with $D=D_{2}$. We also denote

$$
d_{1}^{*}=-\lambda_{1}\left(-g_{1}\left(e^{-k_{0} x}\right)\right), \quad d_{2}^{*}=-\mu_{1}\left(-g_{2}\left(e^{-k_{0} x}\right)\right) .
$$

Nonnegative solutions of (4.1) can be categorized into three classes: (i) The unique trivial solution $\left(u_{1}, u_{2}\right) \equiv(0,0)$, which exists for all $d_{1}$ and $d_{2}$. (ii) Two semitrivial solutions $\left(u_{1}, u_{2}\right)=\left(0, u_{d_{2}}^{*}\right)$ and $\left(u_{1}, u_{2}\right)=\left(u_{d_{1}}^{*}, 0\right)$, the former existing for $d_{2} \in\left(0, d_{2}^{*}\right)$ and the latter existing for $d_{1} \in\left(0, d_{1}^{*}\right)$, where $u_{d_{1}}^{*}, d_{d_{2}}^{*}$ denote the unique steady state for the $u_{1}$ and $u_{2}$ equations, respectively, guaranteed by Theorem 2.1. (iii) The third class are positive solutions $\left(u_{1}, u_{2}\right)$ with $u_{1}(x)>0$ and $u_{2}(x)>0$ in $[0,1]$, which are the most difficult to understand and are the main interest here.

Some necessary conditions for the existence of a positive solution to (4.1) can be easily observed. Suppose that $\left(u_{1}, u_{2}\right)$ is a positive solution of (4.1). Then from the equation for $u_{1}$ we obtain

$$
-d_{1}=\lambda_{1}\left(-g_{1}\left(e^{-k_{0} x-\int_{0}^{x}\left(k_{1} u_{1}+k_{2} u_{2}\right) d y}\right)\right) \in\left(\lambda_{1}\left(-g_{1}\left(e^{-k_{0} x}\right)\right), \lambda_{1}(0)\right)=\left(-d_{1}^{*}, 0\right) .
$$

That is $d_{1} \in\left(0, d_{1}^{*}\right)$. Similarly from the equation for $u_{2}$ we deduce $d_{2} \in\left(0, d_{2}^{*}\right)$. Thus for (4.1) to possess a positive solution we necessarily have

$$
0<d_{1}<d_{1}^{*}, \quad 0<d_{2}<d_{2}^{*} .
$$

Next we assume (4.2) and use a global bifurcation argument to find sufficient conditions for the existence of positive solutions to (4.1). We will rewrite (4.1) as an abstract equation involving a completely continuous operator. Let $E=C([0,1])$, and let $P$ be the usual positive cone in $E: P=\{u \in E: u(x) \geq 0$ in $[0,1]\}$. We define $A: E \times E \rightarrow E \times E$ by

$$
A\left(u_{1}, u_{2}\right)=\left(A_{1}\left(u_{1}, u_{2}\right), A_{2}\left(u_{1}, u_{2}\right)\right)
$$

where

$$
\begin{gathered}
A_{1}\left(u_{1}, u_{2}\right)=L_{1} \circ G_{1}\left(d_{1}, u_{1}, u_{2}\right), \quad A_{2}\left(u_{1}, u_{2}\right)=L_{2} \circ G_{2}\left(d_{2}, u_{1}, u_{2}\right), \\
G_{1}\left(d_{1}, u_{1}, u_{2}\right)(x)=\left[d_{1}^{*}-d_{1}+g_{1}\left(e^{-k_{0} x-\int_{0}^{x}\left(k_{1} u_{1}+k_{2} u_{2}\right) d y}\right)\right] u_{1}(x),
\end{gathered}
$$

Copyright $@$ by SIAM. Unauthorized reproduction of this article is prohibited. 


$$
G_{2}\left(d_{2}, u_{1}, u_{2}\right)(x)=\left[d_{2}^{*}-d_{2}+g_{2}\left(e^{-k_{0} x-\int_{0}^{x}\left(k_{1} u_{1}+k_{2} u_{2}\right) d y}\right)\right] u_{2}(x),
$$

and for $i=1,2, L_{i}$ is the solution operator for the problem

$$
-D_{i} u_{x x}+d_{i}^{*} u=f_{i}(x), \quad u_{x}(0)=u_{x}(1)=0,
$$

namely, $u=L_{i}\left(f_{i}\right)$. It is easily seen that $\left(u_{1}, u_{2}\right)$ solves (4.1) if and only if $\left(u_{1}, u_{2}\right)=$ $A\left(u_{1}, u_{2}\right)$.

By standard elliptic regularity theory we know that $A: E \times E \rightarrow E \times E$ is completely continuous. Moreover, by the strong maximum principle and the fact that (due to $(4.2)$ )

$$
d_{i}^{*}-d_{i}+g_{i}\left(e^{-k_{0} x-\int_{0}^{x}\left(k_{1} u_{1}+k_{2} u_{2}\right) d y}\right)>0 \text { in }[0,1],
$$

we find that if $u_{i} \in P$, then $A_{i}\left(u_{1}, u_{2}\right) \in P$, and if $u_{i} \in \dot{P}:=P \backslash\{0\}$, then $A_{i}\left(u_{1}, u_{2}\right) \in$ $P^{\circ}:=\{u \in P: u(x)>0$ in $[0,1]\}$. Thus we have

$$
\begin{aligned}
& A(P \times P) \subset P \times P, A(\dot{P} \times \dot{P}) \subset P^{\circ} \times P^{\circ}, \\
& A(\dot{P} \times P) \subset P^{\circ} \times P, A(P \times \dot{P}) \subset P \times P^{\circ} .
\end{aligned}
$$

To apply the bifurcation argument, we will fix $d_{1} \in\left(0, d_{1}^{*}\right)$ and use $d_{2}$ as the bifurcation parameter. To stress the dependence of $A\left(u_{1}, u_{2}\right)$ on $d_{2}$, we rewrite it as $A\left(d_{2}, u_{1}, u_{2}\right)$. We will examine how a positive solution branch $\Gamma=\left\{\left(d_{2}, u_{1}, u_{2}\right)\right\} \subset$ $R^{1} \times P \times P$ bifurcates from the semitrivial solution branches $\Gamma_{1}=\left\{\left(d_{2}, u_{d_{1}}^{*}, 0\right)\right.$ : $\left.d_{2} \in R^{1}\right\}$ and $\Gamma_{2}=\left\{\left(d_{2}, 0, u_{d_{2}}^{*}\right): d_{2} \in\left(0, d_{2}^{*}\right)\right\}$. To this end, the Fréchet derivative of $A\left(d_{2}, u_{1}, u_{2}\right)$, with respect to $\left(u_{1}, u_{2}\right)$ at $\left(u_{d_{1}}^{*}, 0\right)$ and at $\left(0, u_{d_{2}}^{*}\right)$, and the associated eigenvalue problems play a crucial role. We will denote these derivatives by $A_{\left(u_{1}, u_{2}\right)}^{\prime}\left(d_{2}, u_{d_{1}}^{*}, 0\right)$ and $A_{\left(u_{1}, u_{2}\right)}^{\prime}\left(d_{2}, 0, u_{d_{2}}^{*}\right)$, respectively, and the associated eigenvalue problems are

$$
A_{\left(u_{1}, u_{2}\right)}^{\prime}\left(d_{2}, u_{d_{1}}^{*}, 0\right)\left(h_{1}, h_{2}\right)^{T}=\xi\left(h_{1}, h_{2}\right)^{T}
$$

and

$$
A_{\left(u_{1}, u_{2}\right)}^{\prime}\left(d_{2}, 0, u_{d_{2}}^{*}\right)\left(h_{1}, h_{2}\right)^{T}=\eta\left(h_{1}, h_{2}\right)^{T} .
$$

Here we use $\left(h_{1}, h_{2}\right)^{T}$ to denote the transpose of the row matrix $\left(h_{1}, h_{2}\right)$. A direct calculation shows that $\eta=1$ is an eigenvalue of (2.7) if and only if the following problem has a solution $\left(h_{1}, h_{2}\right) \neq(0,0)$ :

$$
\left\{\begin{aligned}
-D_{1} h_{1}^{\prime \prime}= & {\left[g_{1}\left(\sigma_{2}(x)\right)-d_{1}\right] h_{1}, } & & x \in(0,1) \\
-D_{2} h_{2}^{\prime \prime}= & {\left[g_{2}\left(\sigma_{2}(x)\right)-d_{2}\right] h_{2} } & & \\
& -g_{2}^{\prime}\left(\sigma_{2}(x)\right) u_{d_{2}}^{*}(x) \sigma_{2}(x) \int_{0}^{x}\left[k_{1} h_{1}(y)+k_{2} h_{2}(y)\right] d y, & & x \in(0,1) \\
h_{1}^{\prime}= & h_{2}^{\prime}=0, & & x=0,1
\end{aligned}\right.
$$

where

$$
\sigma_{2}(x)=e^{-k_{0} x-\int_{0}^{x} k_{2} u_{d_{2}}^{*}(y) d y}
$$

Similarly, if we define

$$
\sigma_{1}(x)=e^{-k_{0} x-\int_{0}^{x} k_{1} u_{d_{1}}^{*}(y) d y},
$$

Copyright (c) by SIAM. Unauthorized reproduction of this article is prohibited. 
then $\xi=1$ is an eigenvalue of (4.3) if and only if the following problem has a solution $\left(h_{1}, h_{2}\right) \neq(0,0)$ :

$$
\left\{\begin{aligned}
-D_{1} h_{1}^{\prime \prime}= & {\left[g_{1}\left(\sigma_{1}(x)\right)-d_{1}\right] h_{1} } & & \\
& -g_{1}^{\prime}\left(\sigma_{1}(x)\right) u_{d_{1}}^{*}(x) \sigma_{1}(x) \int_{0}^{x}\left[k_{1} h_{1}(y)+k_{2} h_{2}(y)\right] d y, & & x \in(0,1), \\
-D_{2} h_{2}^{\prime \prime}= & {\left[g_{2}\left(\sigma_{1}(x)\right)-d_{2}\right] h_{2}, } & & x \in(0,1), \\
h_{1}^{\prime}= & h_{2}^{\prime}=0, & & x=0,1 .
\end{aligned}\right.
$$

The following lemma holds the key for solving (4.5) and (4.6).

Lemma 4.1. Let $i \in\{1,2\}$. If $\psi \in C^{2}([0,1])$ satisfies

$$
\left\{\begin{aligned}
-D_{i} \psi^{\prime \prime} & =\left[g_{i}\left(\sigma_{i}(x)\right)-d_{i}\right] \psi-g_{i}^{\prime}\left(\sigma_{i}(x)\right) u_{d_{i}}^{*}(x) \sigma_{i}(x) \int_{0}^{x} k_{i} \psi(y) d y, \quad x \in(0,1), \\
\psi^{\prime}(0) & =\psi^{\prime}(1)=0,
\end{aligned}\right.
$$

then $\psi \equiv 0$.

Proof. We argue indirectly. Suppose $\psi \not \equiv 0$ solves (4.7). We first claim that $\psi(0) \neq 0$. Otherwise, define

$$
\xi(x)=\int_{0}^{x} \psi(y) d y, \eta(x)=\psi^{\prime}(x)
$$

Then $(\xi(x), \psi(x), \eta(x))$ is a solution of the ODE system

$$
\left\{\begin{array}{l}
\xi^{\prime}=\psi \\
\psi^{\prime}=\eta \\
\eta^{\prime}=-D_{i}^{-1}\left[g_{i}\left(\sigma_{i}(x)\right)-d_{i}\right] \psi+D_{i}^{-1} g_{i}^{\prime}\left(\sigma_{i}(x)\right) u_{d_{i}}^{*}(x) \sigma_{i}(x) k_{i} \xi
\end{array}\right.
$$

with initial condition $(\xi(0), \psi(0), \eta(0))=(0,0,0)$. Clearly $(\xi, \psi, \eta) \equiv(0,0,0)$ is the unique solution of this initial value ODE problem. Hence $\psi \equiv 0$, contradicting our assumption that $\psi \not \equiv 0$. This proves our claim that $\psi(0) \neq 0$.

Without loss of generality we may assume that $\psi(0)>0$. Next we claim that $\psi(x)$ changes sign in $(0,1)$. Otherwise $\psi(x) \geq, \not \equiv 0$ in $[0,1]$. Multiply the first equation in (4.7) by $u_{d_{i}}^{*}$ and integrate it over $[0,1]$; we easily deduce

$$
\int_{0}^{1}\left(g_{i}^{\prime}\left(\sigma_{i}(x)\right)\left[u_{d_{i}}^{*}(x)\right]^{2} \sigma_{i}(x) \int_{0}^{x} k_{i} \psi(y) d y\right) d x=0
$$

But the integrand function of $x$ is clearly nonnegative and not identically zero in $[0,1]$. Hence the integral should be positive. This contradiction shows that $\psi(x)$ changes sign in $(0,1)$.

Let $x_{0} \in(0,1)$ be the first zero of $\psi(x)$, namely, $\psi(x)>0$ in $\left[0, x_{0}\right)$ and $\psi\left(x_{0}\right)=0$. We now consider the eigenvalue problem

$$
-D_{i} \phi^{\prime \prime}=\left[g_{i}\left(\sigma_{i}(x)\right)-d_{i}\right] \phi+\lambda \phi \text { in }\left(0, x_{0}\right), \phi^{\prime}(0)=\phi\left(x_{0}\right)=0
$$

We claim that the first eigenvalue $\lambda_{1}$ of this problem is positive. Indeed, let $\phi_{1}$ be a positive eigenfunction corresponding to $\lambda_{1}$. By the Hopf boundary lemma, $\phi_{1}^{\prime}\left(x_{0}\right)<0$. Multiplying the first equation in (4.8) (with $\lambda=\lambda_{1}, \phi=\phi_{1}$ ) by $u_{d_{i}}^{*}$ and integrating 
over $\left[0, x_{0}\right]$ we obtain

$$
\begin{aligned}
\int_{0}^{x_{0}}\left[g_{i}\left(\sigma_{i}\right)-d_{i}\right] \phi_{1} u_{d_{i}}^{*}+\lambda_{1} \int_{0}^{x_{0}} \phi_{1} u_{d_{i}}^{*} & =-D_{i} \int_{0}^{x_{0}} \phi_{1}^{\prime \prime} u_{d_{i}}^{*} \\
& =-D_{i} \phi_{1}^{\prime}\left(x_{0}\right) u_{d_{i}}^{*}\left(x_{0}\right)-D_{i} \int_{0}^{x_{0}}\left(u_{d_{i}}^{*}\right)^{\prime \prime} \phi_{1} \\
& >\int_{0}^{x_{0}}\left[g_{i}\left(\sigma_{i}\right)-d_{i}\right] \phi_{1} u_{d_{i}}^{*} .
\end{aligned}
$$

Hence $\lambda_{1} \int_{0}^{x_{0}} \phi_{1} u_{d_{i}}^{*}>0$, which gives $\lambda_{1}>0$, as claimed.

To obtain the desired contradiction, we now multiply the first equation in (4.8) (with $\lambda=\lambda_{1}, \phi=\phi_{1}$ ) by $\psi$ and then integrate it over $\left[0, x_{0}\right]$, which gives us

$$
\begin{aligned}
& \int_{0}^{x_{0}}\left[g_{i}\left(\sigma_{i}\right)-d_{i}\right] \phi_{1} \psi+\lambda_{1} \int_{0}^{x_{0}} \phi_{1} \psi \\
& =-D_{i} \int_{0}^{x_{0}} \phi_{1}^{\prime \prime} \psi=-D_{i} \int_{0}^{x_{0}} \psi^{\prime \prime} \phi_{1} \\
& =\int_{0}^{x_{0}}\left[g_{i}\left(\sigma_{i}\right)-d_{i}\right] \phi_{1} \psi-\int_{0}^{x_{0}}\left[g_{i}^{\prime}\left(\sigma_{i}\right) \sigma_{i} u_{d_{i}}^{*} \int_{0}^{x} k_{i} \psi d y\right] \phi_{1} d x .
\end{aligned}
$$

It follows that

$$
\lambda_{1} \int_{0}^{x_{0}} \phi_{1} \psi=-\int_{0}^{x_{0}}\left[g_{i}^{\prime}\left(\sigma_{i}\right) \sigma_{i} u_{d_{i}}^{*} \int_{0}^{x} k_{i} \psi d y\right] \phi_{1} d x .
$$

Since $\lambda_{1}>0$ and $\psi(x)>0$ in $\left[0, x_{0}\right)$, the left side of the above identity is positive. However, the bracketed integrand function on the right side of (4.9) is nonnegative, and hence the right side of (4.9) is not positive. This contradiction completes the proof.

Using Lemma 4.1, we can easily prove the following result.

Lemma 4.2. Problem (4.5) has a solution $\left(h_{1}, h_{2}\right) \neq(0,0)$ if and only if $h_{1} \neq 0$ and

$$
-D_{1} h_{1}^{\prime \prime}=\left[g_{1}\left(\sigma_{2}(x)\right)-d_{1}\right] h_{1}, h_{1}^{\prime}(0)=h_{1}^{\prime}(1)=0 .
$$

Moreover, with $h_{1}$ given, $h_{2}$ can be uniquely solved from the second equation in (4.5) together with the Neumann boundary conditions.

Similarly, (4.6) has a solution $\left(h_{1}, h_{2}\right) \neq(0,0)$ if and only if $h_{2} \neq 0$ and

$$
-D_{2} h_{2}^{\prime \prime}=\left[g_{2}\left(\sigma_{1}(x)\right)-d_{2}\right] h_{2}, h_{2}^{\prime}(0)=h_{2}^{\prime}(1)=0 .
$$

Moreover, with $h_{2}$ given, $h_{1}$ can be uniquely solved from the first equation in (4.6) together with the Neumann boundary conditions.

Proof. We consider only the statement for (4.5); the proof of that for (4.6) is analogous. Let $\left(h_{1}, h_{2}\right)$ solve (4.5). If $h_{1}=0$, then by Lemma 4.1 we deduce $h_{2}=0$. Suppose now $h_{1} \neq 0$. Then we can apply the Fredholm alternative for compact operators and Lemma 4.1 to conclude that the second equation in (4.5) together with the Neumann boundary conditions is uniquely solvable for any given $h_{1}$.

Recall that

$$
\sigma_{2}(x)=\sigma_{2}\left(d_{2}, x\right)=e^{-k_{0} x-\int_{0}^{x} k_{2} u_{d_{2}}^{*}(y) d y}
$$

Copyright $@$ ㅇ by SIAM. Unauthorized reproduction of this article is prohibited. 
and

$$
\sigma_{1}(x)=\sigma_{1}\left(d_{1}, x\right)=e^{-k_{0} x-\int_{0}^{x} k_{1} u_{d_{1}}^{*}(y) d y} .
$$

By Theorem 2.1(iv), we know that $d_{2} \rightarrow \sigma_{2}\left(d_{2}, x\right)$ is strictly increasing for $x \in(0,1]$. This implies that

$$
\lambda\left(d_{2}\right):=-\lambda_{1}\left(-g_{1}\left(\sigma_{2}\left(d_{2}, \cdot\right)\right)\right)
$$

is strictly increasing for $d_{2} \in\left(0, d_{2}^{*}\right)$. By Theorem 2.1(i), we know that $\lambda\left(d_{2}\right)$ is continuous. Since $u_{d_{2}}^{*}(x) \rightarrow 0$ uniformly in $[0,1]$ as $d_{2} \rightarrow d_{2}^{*}$ and $u_{d_{2}}^{*}(x) \rightarrow \infty$ uniformly in $[0,1]$ as $d_{2} \rightarrow 0$, we easily see that

$$
\lim _{d_{2} \rightarrow 0} \lambda\left(d_{2}\right)=0, \quad \lim _{d_{2} \rightarrow d_{2}^{*}} \lambda\left(d_{2}\right)=d_{1}^{*} .
$$

Therefore for any given $d_{1} \in\left(0, d_{1}^{*}\right)$, there exists a unique $\hat{d}_{2}=\hat{d}_{2}\left(d_{1}\right) \in\left(0, d_{1}^{*}\right)$ such that

$$
\lambda\left(\hat{d}_{2}\right)=d_{1}
$$

Let us also introduce

$$
\tilde{d}_{2}=\tilde{d}_{2}\left(d_{1}\right):=-\mu_{1}\left(-g_{2}\left(\sigma_{1}\left(d_{1}, \cdot\right)\right)\right) .
$$

It is easily seen that $\tilde{d}_{2} \in\left(0, d_{2}^{*}\right)$.

We are now ready to state and prove our main result of this section.

Theorem 4.3. For fixed $d_{1} \in\left(0, d_{1}^{*}\right)$ let $\hat{d}_{2}$ and $\tilde{d}_{2}$ be defined as above. Then (4.1) has a positive solution $\left(u_{1}, u_{2}\right)$ if $d_{2}$ lies between $\hat{d}_{2}$ and $\tilde{d}_{2}$. Moreover, there exists a branch of positive solutions $\Gamma=\left\{\left(d_{2}, u_{1}, u_{2}\right)\right\} \subset R^{1} \times E \times E$ that meets the semitrivial solution branch $\Gamma_{1}=\left\{\left(d_{2}, u_{d_{1}}^{*}, 0\right): d_{2} \in R^{1}\right\}$ precisely at $\left(\tilde{d}_{2}, u_{d_{1}}^{*}, 0\right)$, and meets the semitrivial solution branch $\Gamma_{2}=\left\{\left(d_{2}, 0, u_{d_{2}}^{*}\right): d_{2} \in\left(0, d_{2}^{*}\right)\right\}$ precisely at $\left(\hat{d}_{2}, 0, u_{\hat{d}_{2}}^{*}\right)$. More accurately, $\Gamma$ is a connected set in $R^{1} \times E \times E$ such that $\Gamma \cap \Gamma_{1}=\left\{\left(\tilde{d}_{2}, u_{d_{1}}^{*}, 0\right)\right\}$, $\Gamma \cap \Gamma_{2}=\left\{\left(\hat{d}_{2}, 0, u_{\hat{d}_{2}}^{*}\right)\right\}$, and $\Gamma \backslash\left\{\left(\tilde{d}_{2}, u_{d_{1}}^{*}, 0\right),\left(\hat{d}_{2}, 0, u_{\hat{d}_{2}}^{*}\right)\right\}$ consists of positive solutions of (4.1).

Proof. For clarity we divide the proof into four steps.

Step 1 . We show that for any small $\delta>0$ and fixed $d_{1} \in\left(0, d_{1}^{*}\right)$, there exists $C$ such that any positive solution $\left(u_{1}, u_{2}\right)$ of (4.1) with $d_{2} \in\left[\delta, d_{2}^{*}-\delta\right]$ satisfies

$$
\left\|u_{1}\right\|_{\infty}+\left\|u_{2}\right\|_{\infty}<C
$$

Otherwise we can find a small $\delta>0$ and a sequence $d_{2}^{n} \in\left[\delta, d_{2}^{*}-\delta\right]$ such that (4.1) with $d_{2}=d_{2}^{n}$ has a positive solution $\left(u_{1}^{n}, u_{2}^{n}\right)$ satisfying

$$
\lim _{n \rightarrow \infty}\left(\left\|u_{1}^{n}\right\|_{\infty}+\left\|u_{2}^{n}\right\|_{\infty}\right)=\infty .
$$

By passing to a subsequence, we have either $\left\|u_{2}^{n}\right\|_{\infty} \rightarrow \infty$ or $\left\|u_{1}^{n}\right\|_{\infty} \rightarrow \infty$. In the first case, we define $\hat{u}_{n}=u_{2}^{n} /\left\|u_{2}^{n}\right\|_{\infty}$. Then from the equation for $u_{2}^{n}$ we deduce

$$
-D_{2} \hat{u}_{n}^{\prime \prime}=\left[g_{2}\left(f_{n}(x)\right)-d_{2}^{n}\right] \hat{u}_{n}, \hat{u}_{n}^{\prime}(0)=\hat{u}_{n}^{\prime}(1)=0,
$$

where

$$
f_{n}(x)=e^{-k_{0} x-\int_{0}^{x}\left(k_{1} u_{1}^{n}+k_{2} u_{2}^{n}\right) d y} .
$$

Copyright $@$ by SIAM. Unauthorized reproduction of this article is prohibited. 
Since $\left\|\hat{u}_{n}\right\|_{\infty}=1$ and the right side of the first equation in (4.13) has a bound in $L^{\infty}([0,1])$ that is independent of $n$, by standard elliptic regularity we know that $\left\{\hat{u}_{n}\right\}$ is precompact in $C^{1}([0,1])$. Hence by passing to a subsequence we may assume that $\hat{u}_{n} \rightarrow \hat{u}$ in $C^{1}([0,1])$. Since $0<f_{n}(x) \leq 1$ and $\delta \leq d_{2}^{n} \leq d_{2}^{*}-\delta$, by passing to a subsequence we may assume that $f_{n} \rightarrow f$ weakly in $L^{2}([0,1])$ and $d_{2}^{n} \rightarrow d_{2}^{\infty}$, with $0 \leq f \leq 1$ and $d_{2}^{\infty} \in\left[\delta, d_{2}^{*}-\delta\right]$. Since $g_{2}$ is $C^{1}$, we easily see that $g_{2}\left(f_{n}\right) \rightarrow g_{2}(f)$ weakly in $L^{2}([0,1])$. Thus $\hat{u}$ is a weak solution to

$$
-D_{2} \hat{u}^{\prime \prime}=\left[g_{2}(f(x))-d_{2}^{\infty}\right] \hat{u}, \quad \hat{u}^{\prime}(0)=\hat{u}^{\prime}(1)=0 .
$$

Clearly $\hat{u} \geq 0$ and $\|\hat{u}\|_{\infty}=1$. Thus we can apply the strong maximum principle to conclude that $\hat{u}(x)>0$ in $[0,1]$. This implies that $u_{2}^{n}(x) \rightarrow \infty$ uniformly in $[0,1]$, and hence $f \equiv 0, g_{2}(f) \equiv 0$. Thus $\hat{u}$ is a positive solution of

$$
-D_{2} \hat{u}^{\prime \prime}=-d_{2}^{\infty} \hat{u}, \quad \hat{u}^{\prime}(0)=\hat{u}^{\prime}(1)=0 .
$$

This implies that $-d_{2}^{\infty}=\mu_{1}(0)=0$, a contradiction to $d_{2}^{\infty} \in\left[\delta, d_{2}^{*}-\delta\right]$.

If $\left\|u_{1}^{n}\right\|_{\infty} \rightarrow \infty$, then we can use the equation for $u_{1}^{n}$ and a similar argument to reach a contradiction. This completes the proof of Step 1.

Step 2. We show that if $\delta>0$ is small enough, then (4.1) has no positive solution if $d_{2} \notin\left(\delta, d_{2}^{*}-\delta\right)$.

Otherwise, due to (4.2), we can find $d_{2}^{n} \downarrow 0$ or $d_{2}^{n} \uparrow d_{2}^{*}$ and a positive solution $\left(u_{1}^{n}, u_{2}^{n}\right)$ of (4.1) with $d_{2}=d_{2}^{n}$. In the first case we define $\hat{u}_{n}$ and $f_{n}$ as in Step 1 above and find by the same reasoning that by passing to a subsequence $\hat{u}_{n} \rightarrow \hat{u}$ in $C^{1}([0,1])$, $f_{n} \rightarrow f$ and $g_{2}\left(f_{n}\right) \rightarrow g_{2}(f)$ weakly in $L^{2}([0,1])$, and $\hat{u}$ is a positive solution to

$$
-D_{2} \hat{u}^{\prime \prime}=g_{2}(f) \hat{u}, \quad \hat{u}^{\prime}(0)=\hat{u}^{\prime}(1)=0 .
$$

Integrating the first equation for $x$ over $[0,1]$, we deduce $\int_{0}^{1} g_{2}(f) \hat{u} d x=0$. Since $\hat{u}>0$ in $[0,1]$ and $g_{2}(f(x)) \geq 0$ in $[0,1]$, the above identity implies that $g_{2}(f(x))=0$ a.e. in $[0,1]$. It follows that $f(x)=0$ a.e. in $[0,1]$.

Now we define $\hat{v}_{n}=u_{1}^{n} /\left\|u_{1}^{n}\right\|_{\infty}$ and obtain from the equation for $u_{1}^{n}$ that

$$
-D_{1} \hat{v}_{n}=\left[g_{1}\left(f_{n}(x)\right)-d_{1}\right] \hat{v}_{n}, \quad \hat{v}_{n}^{\prime}(0)=\hat{v}_{n}^{\prime}(1)=0 .
$$

As before by elliptic regularity, subject to passing to a subsequence, $\hat{v}_{n} \rightarrow \hat{v}$ in $C^{1}([0,1])$ and $g_{2}\left(f_{n}\right) \rightarrow g_{1}(f)=0$ weakly in $L^{2}([0,1])$, and $\hat{v}$ is a positive solution to

$$
-D_{1} \hat{v}^{\prime \prime}=-d_{1} \hat{v}, \quad \hat{v}^{\prime}(0)=\hat{v}^{\prime}(1)=0 .
$$

This implies $d_{1}=0$, a contradiction to our assumption $d_{1} \in\left(0, d_{1}^{*}\right)$.

Next we consider the case $d_{2}^{n} \rightarrow d_{2}^{*}$. We define $\hat{u}_{n}, \hat{v}_{n}$, and $f_{n}$ as above. By the same argument we know that by passing to a subsequence, $\hat{u}_{n} \rightarrow \hat{u}$ and $\hat{v}_{n} \rightarrow \hat{v}$ in $C^{1}([0,1]), f_{n} \rightarrow f$ and $g_{i}\left(f_{n}\right) \rightarrow g_{i}(f)$ weakly in $L^{2}([0,1])$, and $\hat{u}, \hat{v}$ are positive solutions to

$$
-D_{2} \hat{u}^{\prime \prime}=\left[g_{2}(f)-d_{2}^{*}\right] \hat{u}, \quad \hat{u}^{\prime}(0)=\hat{u}^{\prime}(1)=0
$$

and

$$
-D_{1} \hat{v}^{\prime \prime}=\left[g_{1}(f)-d_{1}\right] \hat{v}, \quad \hat{v}^{\prime}(0)=\hat{v}^{\prime}(1)=0,
$$

respectively. 
Let us now look at the sequence $\left\{\left\|u_{1}^{n}\right\|_{\infty}\right\}$. If this sequence is not bounded, then by passing to a subsequence we have $\left\|u_{1}^{n}\right\|_{\infty} \rightarrow \infty$ and hence $u_{1}^{n}=\left\|u_{1}^{n}\right\|_{\infty} \hat{v}_{n} \rightarrow \infty$ uniformly in $[0,1]$. This implies that $f \equiv 0$, and hence (4.15) becomes

$$
-D_{1} \hat{v}^{\prime \prime}=-d_{1} \hat{v}, \quad \hat{v}^{\prime}(0)=\hat{v}^{\prime}(1)=0,
$$

which implies $d_{1}=0$, a contradiction. Thus $\left\{\left\|u_{1}^{n}\right\|_{\infty}\right\}$ is bounded. For the same reason, $\left\{\left\|u_{2}^{n}\right\|_{\infty}\right\}$ is bounded. So we may assume that

$$
\left\|u_{1}^{n}\right\|_{\infty} \rightarrow \alpha_{1} \geq 0, \quad\left\|u_{2}^{n}\right\|_{\infty} \rightarrow \alpha_{2} \geq 0 .
$$

It then follows that

$$
f_{n}(x) \rightarrow e^{-k_{0} x-\int_{0}^{x}\left(k_{1} \alpha_{1} \hat{v}+k_{2} \alpha_{2} \hat{u}\right) d y} \text { uniformly in }[0,1] .
$$

Thus

$$
f(x)=e^{-k_{0} x-\int_{0}^{x}\left(k_{1} \alpha_{1} \hat{v}+k_{2} \alpha_{2} \hat{u}\right) d y} \leq e^{-k_{0} x},
$$

with equality holding for all $x \in[0,1]$ if and only if $\alpha_{1}=\alpha_{2}=0$. It follows that $g_{2}(f(x)) \leq g_{2}\left(e^{-k_{0} x}\right)$, with equality holding for all $x \in[0,1]$ if and only if $\alpha_{1}=\alpha_{2}=0$. From this and (4.14) we deduce

$$
d_{2}^{*}=-\mu_{1}\left(-g_{2}(f)\right) \leq-\mu_{1}\left(-g_{2}\left(e^{-k_{0} x}\right)\right),
$$

with equality holding if and only if $\alpha_{1}=\alpha_{2}=0$. Thus in view of the definition of $d_{2}^{*}$, we necessarily have $\alpha_{1}=\alpha_{2}=0$ and thus $f(x)=e^{-k_{0} x}$. We now use (4.15) and find

$$
d_{1}=-\lambda_{1}\left(-g_{1}(f)\right)=-\lambda_{1}\left(-g_{1}\left(e^{-k_{0} x}\right)\right) .
$$

That is, $d_{1}=d_{1}^{*}$, a contradiction to our assumption on $d_{1}$. This completes our proof of Step 2.

Step 3. Global bifurcation analysis. Let us now fix $\delta>0$ small enough such that (4.1) has no positive solution for $d_{2} \notin \Lambda:=\left(\delta, d_{2}^{*}-\delta\right)$, and $\tilde{d}_{2}, \hat{d}_{2} \in \Lambda$. Then define $\Omega=\Lambda \times U \times V$ with

$$
U=\left\{u_{1} \in P:\left\|u_{1}\right\|_{\infty}<C\right\}, \quad V=\left\{u_{2} \in P:\left\|u_{2}\right\|_{\infty}<C\right\},
$$

where $C>0$ is large enough such that (4.12) holds and $\left\|u_{d_{1}}^{*}\right\|_{\infty}<C$.

We are going to apply the global bifurcation result of [5], namely, [5, Theorem 2.3], to the operator

$$
\left(d_{2}, u_{1}, u_{2}\right) \in \Omega \rightarrow A\left(d_{2}, u_{1}, u_{2}\right) \in P \times P .
$$

It is easily seen that the conditions $\left(\mathrm{I}^{\prime}\right),\left(\mathrm{II}^{\prime}\right)$, and $\left(\mathrm{III}^{\prime}\right)$ in that theorem are satisfied, with $T=\left\{\left(u_{d_{1}}^{*}, 0\right)\right\}$. By that theorem, if for some small $\epsilon>0, A\left(d_{2}, u_{1}, u_{2}\right)=\left(u_{1}, u_{2}\right)$ has no bifurcation value in $\left(\tilde{d}_{2}-\epsilon, \tilde{d}_{2}\right) \cup\left(\tilde{d}_{2}, \tilde{d}_{2}+\epsilon\right)$, and

$$
\operatorname{index}_{P \times P}\left(A(\lambda, \cdot),\left(u_{d_{1}}^{*}, 0\right)\right) \neq \operatorname{index}_{P \times P}\left(A(\mu, \cdot),\left(u_{d_{1}}^{*}, 0\right)\right)
$$

for $\lambda \in\left(\tilde{d}_{2}-\epsilon, \tilde{d}_{2}\right), \mu \in\left(\tilde{d}_{2}, \tilde{d}_{2}+\epsilon\right)$, then

(i) $d_{2}=\tilde{d}_{2}$ is a bifurcation value;

(ii) there is a connected set $\Sigma \subset \bar{\Sigma}_{*}$, where $\Sigma_{*}=\left\{\left(d_{2}, u_{1}, u_{2}\right) \in \Omega: A\left(d_{2}, u_{1}, u_{2}\right)=\right.$ $\left.\left(u_{1}, u_{2}\right), u_{2} \neq 0\right\}$, such that $\Sigma$ contains the point $\left(\tilde{d}_{2}, u_{d_{1}}^{*}, 0\right)$ and 
(a) a point $\left(\lambda_{0}, u_{d_{1}}^{*}, 0\right)$ with $\lambda_{0} \neq \tilde{d}_{2}$, or

(b) a point in $\partial \Omega$, or

(c) a point in $\Lambda \times(0,0)$,

where

$\partial \Omega:=\left\{\left(d_{2}, u_{1}, u_{2}\right) \in \bar{\Omega}: d_{2}=\delta\right.$, or $d_{2}=d_{2}^{*}-\delta$, or $\left\|u_{1}\right\|_{\infty}=C$, or $\left.\left\|u_{2}\right\|_{\infty}=C\right\}$.

By Lemma 4.2 we find that for $\epsilon>0$ small and $d_{2} \in\left[\tilde{d}_{2}-\epsilon, \tilde{d}_{2}+\epsilon\right] \backslash\left\{\tilde{d}_{2}\right\}$, $\left(u_{d_{1}}^{*}, 0\right)$ is a nondegenerate solution of (4.1). Thus there is no bifurcation value in $\left[\tilde{d}_{1}-\epsilon, \tilde{d}_{2}+\epsilon\right] \backslash\left\{\tilde{d}_{2}\right\}$. It follows that the fixed point indices (with respect to the cone $P \times P)$ in (4.16) are well-defined and are independent of $\lambda$ and $\mu$ in their respective given ranges.

Suppose for the moment that (4.16) holds; we now analyze the connected set $\Sigma$. We first observe that $\Sigma \cap \Gamma_{1}=\left\{\left(\tilde{d}_{2}, u_{d_{1}}^{*}, 0\right)\right\}$. Indeed, suppose $\left(\lambda, u_{d_{1}}^{*}, 0\right) \in \Sigma \cap \Gamma_{1}$. Then we can find a sequence of points $\left(d_{2}^{n}, u_{1}^{n}, u_{2}^{n}\right) \in \Sigma_{*}$ that converges to $\left(\lambda, u_{d_{1}}^{*}, 0\right)$ in $R^{1} \times E \times E$. Using the equation for $u_{2}^{n}$, we obtain

$$
-d_{2}^{n}=\mu_{1}\left(-g_{2}\left(e^{-k_{0} x-\int_{0}^{x}\left(k_{1} u_{1}^{n}+k_{2} u_{2}^{n}\right) d y}\right)\right) \rightarrow \mu_{1}\left(-g_{2}\left(e^{-k_{0} x-\int_{0}^{x} k_{1} u_{d_{1}}^{*} d y}\right)\right)=-\tilde{d}_{2} .
$$

Hence $\lambda=\tilde{d}_{2}$. This proves $\Sigma \cap \Gamma_{1}=\left\{\left(\tilde{d}_{2}, u_{d_{1}}^{*}, 0\right)\right\}$, which implies that alternative (a) cannot occur.

We now show that alternative (c) does not happen either. Otherwise, we can find a sequence of points $\left(d_{2}^{n}, u_{1}^{n}, u_{2}^{n}\right) \in \Sigma_{*}$ that converges to $(\lambda, 0,0) \in \Lambda \times(0,0)$. Again we use the equation for $u_{2}^{n}$ and obtain

$$
-d_{2}^{n}=\mu_{1}\left(-g_{2}\left(e^{-k_{0} x-\int_{0}^{x}\left(k_{1} u_{1}^{n}+k_{2} u_{2}^{n}\right) d y}\right)\right) \rightarrow \mu_{1}\left(-g_{2}\left(e^{-k_{0} x}\right)\right)=-d_{2}^{*} .
$$

Thus $d_{2}^{*}=\lambda \in \Lambda=\left(\delta, d_{2}^{*}-\delta\right)$, which is a contradiction. Hence (c) cannot occur.

So alternative (b) necessarily happens. But by our choice of $\delta$ and $C$ used in the definition of $\Omega$, no positive solution $\left(d_{2}, u_{1}, u_{2}\right)$ belongs to $\partial \Omega$. Thus necessarily $\Sigma \cap \partial \Omega$ consists of semitrivial solutions. Since $\Sigma \cap \Gamma_{1}=\left\{\left(\tilde{d}_{2}, u_{d_{1}}^{*}, 0\right)\right\}$ and $\tilde{d}_{2} \in \Lambda$, $\left\|u_{d_{1}}^{*}\right\|_{\infty}<C$, the semitrivial solutions in $\Sigma \cap \partial \Omega$ must belong to $\Gamma_{2}$. This shows that $\Sigma$ intersects $\Gamma_{2}$.

We show next that $\Sigma$ has a subset $\Gamma$ which is connected and has the following properties:

$$
\begin{aligned}
& \Gamma \cap \Gamma_{2}=\left\{\left(\hat{d}_{2}, 0, u_{\hat{d}_{2}}^{*}\right)\right\}, \quad \Gamma \cap \Gamma_{1}=\left\{\left(\tilde{d}_{2}, u_{d_{1}}^{*}, 0\right)\right\} \\
& \Gamma \backslash\left\{\left(\tilde{d}_{2}, u_{d_{1}}^{*}, 0\right),\left(\hat{d}_{2}, 0, u_{\hat{d}_{2}}^{*}\right)\right\} \quad \text { consists of positive solutions of }(4.1) .
\end{aligned}
$$

To this end we consider $\Gamma_{*}:=\Sigma \backslash \Gamma_{2}$. By what was proved above, we know that $\left(\tilde{d}_{2}, u_{d_{1}}^{*}, 0\right) \in \Gamma_{*}$. From the connectedness of $\Sigma$ we easily deduce that $\bar{\Gamma}_{*} \cap \Gamma_{2} \neq \emptyset$. Then much as before we can show that $\bar{\Gamma}_{*} \cap \Gamma_{2}=\left\{\left(\hat{d}_{2}, 0, u_{\hat{d}_{2}}^{*}\right)\right\}$. We claim that $\bar{\Gamma}_{*}$ has a connected component $\Gamma$ which contains both $\left(\hat{d}_{2}, 0, u_{\hat{d}_{2}}^{*}\right)$ and $\left(\tilde{d}_{2}, u_{d_{1}}^{*}, 0\right)$. Otherwise, let $\Gamma_{*}^{1}$ be the maximal connected subset of $\bar{\Gamma}_{*}$ containing $\left(\tilde{d}_{2}, u_{d_{1}}^{*}, 0\right)$; then $\Gamma_{*}^{1}$ and $\Sigma \backslash \Gamma_{*}^{1}$ have positive distance to each other, which implies that $\Sigma$ is not connected. This proves that the above-mentioned $\Gamma$ does exist. Much as before we can easily show that $\Gamma \cap \Gamma_{2}=\left\{\left(\hat{d}_{2}, 0, u_{\hat{d}_{2}}^{*}\right)\right\}$ and $\Gamma \cap \Gamma_{1}=\left\{\left(\tilde{d}_{2}, u_{d_{1}}^{*}, 0\right)\right\}$. It follows that $\Gamma \backslash\left\{\left(\tilde{d}_{2}, u_{d_{1}}^{*}, 0\right),\left(\hat{d}_{2}, 0, u_{\hat{d}_{2}}^{*}\right)\right\}$ consists of positive solutions of (4.1).

To complete the proof of the theorem, it remains to prove (4.16). 
Step 4. Fixed point index calculation. We now calculate the fixed point indices appearing in (4.16) by making use of Theorem 2.1 in [4]. Let $B_{1}$ be a small ball in $E$ containing $u_{d_{1}}^{*}$. Since $u_{d_{1}}^{*} \in P^{\circ}$, we may assume that $B_{1} \subset P^{\circ}$. Then by Theorem 2.1 of [4], we have

$$
\operatorname{index}_{P \times P}\left(A\left(d_{2}, \cdot\right),\left(u_{d_{1}}^{*}, 0\right)\right)= \begin{cases}0 & \text { if } r(L)>1, \\ \operatorname{deg}_{P}\left(I-A_{1}(\cdot, 0), B_{1}\right) & \text { if } r(L)<1,\end{cases}
$$

where $L=\left(A_{2}\right)_{u_{2}}^{\prime}\left(u_{d_{1}}^{*}, 0\right)$ and $r(L)$ denotes the spectral radius of the linear operator $L$.

It is easily checked that $r(L)>1$ if $d_{2}<-\mu_{1}\left(-g_{2}\left(\sigma_{1}\right)\right)=\tilde{d}_{2}$, and $r(L)<1$ if $d_{2}>-\mu_{1}\left(-g_{2}\left(\sigma_{1}\right)\right)=\tilde{d}_{2}$. Thus

$$
\operatorname{index}_{P \times P}\left(A\left(d_{2}, \cdot\right),\left(u_{d_{1}}^{*}, 0\right)\right)= \begin{cases}0 & \text { if } d_{2}<\tilde{d}_{2}, \\ \operatorname{deg}_{P}\left(I-A_{1}(\cdot, 0), B_{1}\right) & \text { if } d_{2}>\tilde{d}_{2} .\end{cases}
$$

We show next that

$$
\operatorname{deg}_{P}\left(I-A_{1}(\cdot, 0), B_{1}\right)=1 .
$$

Since $u_{d_{1}}^{*}$ is the only fixed point of $A_{1}(\cdot, 0)$ in $B_{1}$, we clearly have

$$
\operatorname{deg}_{P}\left(I-A_{1}(\cdot, 0), B_{1}\right)=\operatorname{index}_{P}\left(A_{1}(\cdot, 0), u_{d_{1}}^{*}\right) .
$$

We will use a homotopy argument to $A_{1}\left(\lambda, u_{1}, 0\right)=L_{1} \circ G_{1}\left(\lambda, u_{1}, 0\right)$ with $\lambda \in\left[d_{1}, d_{1}^{*}+1\right]$. By Theorem 2.1 we know that for $\lambda \in\left[d_{1}, d_{1}^{*}\right)$ the equation $A_{1}(\lambda, u, 0)=u$ has exactly two solutions in $P$ : The trivial solution $u=0$ and the unique positive solution $u=u_{\lambda}>0$. For $\lambda \in\left[d_{1}^{*}, d_{1}^{*}+1\right]$, there is one solution in $P$ : $u=0$. Moreover, one easily sees that 0 is a linearized stable fixed point of $A_{1}(\lambda, \cdot, 0)$ when $\lambda>d_{1}^{*}$, and it is a linearized unstable fixed point when $\lambda<d_{1}^{*}$. It follows that

$$
\operatorname{index}_{P}\left(A_{1}(\lambda, \cdot, 0), 0\right)=0 \text { for } \lambda<d_{1}^{*}, \quad \text { and this index is } 1 \text { for } \lambda>d_{1}^{*} .
$$

Choose $C_{0}>0$ large enough such that $\left\|u_{\lambda}\right\|_{\infty}<C_{0}$ for $\lambda \in\left[d_{1}, d_{1}^{*}\right)$, and denote $P_{C_{0}}:=\left\{u \in P:\|u\|_{\infty}<C_{0}\right\}$. Then by the homotopy invariance property of the topological degree, we find that $\operatorname{deg}_{P}\left(I-A(\lambda, \cdot, 0), P_{C_{0}}\right)$ is well-defined and its value does not depend on $\lambda$ for $\lambda \in\left[d_{1}, d_{1}^{*}+1\right]$. By the additivity of the topological degree we have

$$
\begin{aligned}
\operatorname{deg}_{P}\left(I-A_{1}(\lambda, \cdot, 0), P_{C_{0}}\right) & =\operatorname{index}_{P}\left(A_{1}(\lambda, \cdot, 0), 0\right)+\operatorname{index}_{P}\left(A_{1}(\lambda, \cdot, 0), u_{\lambda}\right) \\
& =\operatorname{index}_{P}\left(A_{1}(\lambda, \cdot, 0), u_{\lambda}\right)
\end{aligned}
$$

for $\lambda \in\left[d_{1}, d_{1}^{*}\right)$, and

$$
\operatorname{deg}_{P}\left(I-A(\lambda, \cdot, 0), P_{C_{0}}\right)=\operatorname{index}_{P}\left(A_{1}(\lambda, \cdot, 0), 0\right)=1
$$

for $\lambda \in\left(d_{1}^{*}, d_{1}^{*}+1\right]$. It follows that

$$
\operatorname{index}_{P}\left(A_{1}(\lambda, \cdot, 0), u_{\lambda}\right)=1
$$

for $\lambda \in\left[d_{1}, d_{1}^{*}\right)$. Taking $\lambda=d_{1}$ we obtain

$$
\operatorname{deg}_{P}\left(I-A_{1}(\cdot, 0), B_{1}\right)=\operatorname{index}_{P}\left(A_{1}(\cdot, 0), u_{d_{1}}^{*}\right)=1 .
$$

Thus (4.16) holds, and the proof is complete. 
Remark 4.4. The degree calculation methods developed in $[4,6]$ and the bifurcation method of [5] can also be used to study the existence of positive steady states of the phytoplankton model with three or more species.

We note that the global bifurcation arguments in [2] do not seem easily applicable to our problem here. First, in the abstract global bifurcation result in [2] the fixed point index in the entire space is required, which was calculated there based on a good understanding of the eigenvalues of (4.3) with $\xi \neq 1$. This seems difficult to obtain due to the nonlocal terms in our problem. Second, the analysis in [2] also relies on the local bifurcation result of [3], which requires the bifurcation point to correspond to a simple eigenvalue. In contrast, the global bifurcation result of [5] used here involves the fixed point index in the positive cone, which depends on (4.3) with $\xi=1$ and the second equation in (4.6), which are much easier to handle. Moreover, the result of [5] can be used to discuss systems with more than two equations. (The fixed point index calculation in [2] can also be done by using Theorem 3.1 in [5], which avoids the analysis of the eigenvalues of $(4.3)$ with $\xi \neq 1$.)

We complete this section with some further analysis on $\hat{d}_{2}$ and $\tilde{d}_{2}$. We now regard $d_{i} \in\left(0, d_{i}^{*}\right)(i=1,2)$ as fixed and examine the variation of $\hat{d}_{2}$ and $\tilde{d}_{2}$ as $k_{1}$ and $k_{2}$ vary. Let us note that by definition $d_{1}^{*}$ and $d_{2}^{*}$ are independent of $k_{1}$ and $k_{2}$. We now denote the unique positive solution of the equation for $u_{1}$ in (4.1) by $u_{k_{1}}^{\dagger}$. It is easily checked that $u_{k_{1}}^{\dagger}=k_{1} u_{1}^{\dagger}$. Thus

$$
\sigma_{1}(x)=e^{-k_{0} x-\int_{0}^{x} k_{1}^{2} u_{1}^{\dagger} d y}
$$

It follows that

$$
\tilde{d}_{2}=-\mu_{1}\left(-g_{2}\left(\sigma_{1}\right)\right)
$$

is continuous and strictly decreasing in $k_{1}$ and

$$
\lim _{k_{1} \rightarrow 0} \tilde{d}_{2}=d_{2}^{*}, \quad \lim _{k_{1} \rightarrow \infty} \tilde{d}_{2}=0 .
$$

Thus there exists a unique $k_{1}^{*}>0$ such that

$$
\tilde{d}_{2}=d_{2} \text { if } k_{1}=k_{1}^{*}, \quad \tilde{d}_{2}<d_{2} \text { if } k_{1}>k_{1}^{*}, \quad \tilde{d}_{2}>d_{2} \text { if } 0<k_{1}<k_{1}^{*} .
$$

Similarly, if we define

$$
\tilde{d}_{1}=-\lambda_{1}\left(-g_{1}\left(\sigma_{2}\right)\right)
$$

then there exists a unique $k_{2}^{*}>0$ such that

$$
\tilde{d}_{1}=d_{1} \text { if } k_{2}=k_{2}^{*}, \quad \tilde{d}_{1}<d_{1} \text { if } k_{2}>k_{2}^{*}, \quad \tilde{d}_{1}>d_{1} \text { if } 0<k_{2}<k_{2}^{*} .
$$

On the other hand, it is easily seen that

$$
d_{1}<\tilde{d}_{1} \text { and } d_{2}<\tilde{d}_{2} \text { imply } \hat{d}_{2}<d_{2}<\tilde{d}_{2}
$$

and

$$
d_{1}>\tilde{d}_{1} \text { and } d_{2}>\tilde{d}_{2} \text { imply } \hat{d}_{2}>d_{2}>\tilde{d}_{2}
$$


Hence we can use Theorem 4.3 to obtain the following conclusion:

For fixed $d_{i} \in\left(0, d_{i}^{*}\right)(i=1,2)$, if $\left(k_{1}-k_{1}^{*}\right)\left(k_{2}-k_{2}^{*}\right)>0$, then (4.1) has at least one positive solution.

We conjecture that (4.1) has no positive solution when $\left(k_{1}-k_{1}^{*}\right)\left(k_{2}-k_{2}^{*}\right)<0$, and it has a continuum of positive solutions when $k_{1}=k_{1}^{*}$ and $k_{2}=k_{2}^{*}$.

Remark 4.5. It can be shown that when $0<d_{i}<\tilde{d}_{i}(i=1,2)$, the set of positive solutions of (4.1) has topological degree 1 , and if $d_{i}>\tilde{d}_{i}(i=1,2)$, the set of positive solutions of (4.1) has topological degree -1 . The first conclusion can be extended to the $n \geq 3$ species case (by arguments similar to those in [6]).

5. Uniform persistence and extinction for the two-species model. In this section we consider the corresponding parabolic system of (4.1), namely,

$$
\begin{cases}\left(u_{1}\right)_{t}=D_{1}\left(u_{1}\right)_{x x}+\left[g_{1}(I(x, t))-d_{1}\right] u_{1}, & 0<x<1, t>0 \\ \left(u_{2}\right)_{t}=D_{2}\left(u_{2}\right)_{x x}+\left[g_{2}(I(x, t))-d_{2}\right] u_{2}, & 0<x<1, t>0 \\ \left(u_{i}\right)_{x}(0, t)=\left(u_{i}\right)_{x}(1, t)=0, & t>0, i=1,2 \\ u_{i}(x, 0)=u_{i}^{0}(x), & 0<x<1, i=1,2\end{cases}
$$

where $u_{1}^{0}, u_{2}^{0} \in P, g_{1}(I), g_{2}(I)$ satisfy $(2.4)$, and

$$
I(x, t)=e^{-k_{0} x} \exp \left(-\int_{0}^{x}\left[k_{1} u_{1}(y, t)+k_{2} u_{2}(y, t)\right] d y\right),
$$

with $k_{0}, k_{1}, k_{2}>0$.

If the initial data in (5.1) satisfy $u_{1}^{0} \in \dot{P}$ and $u_{2}^{0} \equiv 0$, then from the results in section 3 we have the following:

(i) If $0<d_{1}<d_{1}^{*}$, then $u_{1}(x, t) \rightarrow u_{1}^{*}(x)>0$ as $t \rightarrow \infty, u_{2}(x, t) \equiv 0$.

(ii) If $d_{1} \geq d_{1}^{*}$, then $u_{1}(x, t) \rightarrow 0$ as $t \rightarrow \infty, u_{2}(x, t) \equiv 0$,

where we use $u_{i}^{*}$ to denote $u_{d_{i}}^{*}$ for convenience.

Similarly, if $u_{2}^{0} \in \dot{P}$ and $u_{1}^{0} \equiv 0$, then we have the following:

(i) If $0<d_{2}<d_{2}^{*}$, then $u_{2}(x, t) \rightarrow u_{2}^{*}(x)>0$ as $t \rightarrow \infty, u_{1}(x, t) \equiv 0$.

(ii) If $d_{2} \geq d_{2}^{*}$, then $u_{2}(x, t) \rightarrow 0$ as $t \rightarrow \infty, u_{1}(x, t) \equiv 0$.

Next we consider the case that $u_{i}^{0} \in \dot{P}$ for $i=1,2$. By standard arguments one sees that (5.1) has a unique solution $\left(u_{1}, u_{2}\right)$ and $u_{i}(x, t)>0$ for $t>0$ and $x \in[0,1]$. Since

$$
\left(u_{i}\right)_{t} \leq D_{i}\left(u_{i}\right)_{x x}+\left[g_{i}(1)-d_{i}\right] u_{i}
$$

one can use the comparison principle to see that $u_{i}$ does not blow up in finite time, and hence it is defined for all $t>0$. We show next that it is uniformly bounded for all $t>0$. Indeed, since $g_{i}(I) \leq \sigma I$ for some $\sigma>0$ and $I \in[0,1]$, we have, for $i=1,2$,

$$
\left(u_{i}\right)_{t} \leq D_{i}\left(u_{i}\right)_{x x}+\left[\sigma e^{-k_{i} \int_{0}^{x} u_{i} d y}-d_{i}\right] u_{i} .
$$

Hence by the argument used in the proof of Lemma 3.2 we deduce that for some $C>0$,

$$
0 \leq u_{i}(x, t) \leq C \text { for all } x \in[0,1] \text { and } t>0
$$


Theorem 5.1 (uniform persistence). If $0<d_{1}<\tilde{d}_{1}$ and $0<d_{2}<\tilde{d}_{2}$, then the system (5.1) with initial data $u_{i}^{0} \in \dot{P}(i=1,2)$ is uniformly persistent: There exists $\epsilon_{0}>0$ such that

$$
\liminf _{t \rightarrow \infty}\left\|u_{1}(\cdot, t)\right\|_{\infty} \geq \epsilon_{0}, \quad \liminf _{t \rightarrow \infty}\left\|u_{2}(\cdot, t)\right\|_{\infty} \geq \epsilon_{0} .
$$

Proof. We apply a general result of Hale and Waltman, namely, Theorem 4.1 in [11]. We note that (5.1) generates a semigroup (more often called a semiflow) $T(t)$ on $P \times P$, and $T(t)$ is compact for $t>0$. By the results of section 3 (for semitrivial solutions) and (5.4) (for positive solutions), $T(t)$ is point dissipative in $P \times P$.

Let

$$
X^{0}=\dot{P} \times \dot{P} \quad \text { and } \quad X=\overline{X^{0}}=P \times P .
$$

Then $X^{0}$ is invariant and relatively open in $X$, and $\partial X^{0}=(P \times\{0\}) \cup(\{0\} \times P)$ is also invariant. From Theorem 3.3 , the rest point $M_{1}=\left(u_{1}^{*}, 0\right)$ attracts $\left(u_{1}^{0}, 0\right)$ with $u_{1}^{0} \in \dot{P}$, and $M_{2}=\left(0, u_{2}^{*}\right)$ attracts $\left(0, u_{2}^{0}\right)$ with $u_{2}^{0} \in \dot{P}$. The omega limit sets of the semiflow on $\partial X^{0}$, denoted by $\tilde{A}_{\partial}$, are given by $\tilde{A}_{\partial}=\left\{M_{0}, M_{1}, M_{2}\right\}$, where $M_{0}=(0,0)$. Now $M=\left\{M_{0}, M_{1}, M_{2}\right\}$ is a covering of $\tilde{A}_{\partial}$. From (5.2) and (5.3), $M_{0}$ is a repeller, $M$ contains no cycle, and this covering is isolated. To apply Theorem 4.1 in [11], it remains to check that the stable set of $M_{i}$, denoted by $W^{s}\left(M_{i}\right)$, does not intersect $X^{0}$, that is, $W^{s}\left(M_{i}\right) \cap X^{0}=\emptyset$.

Suppose that $\left(u_{1}^{0}, u_{2}^{0}\right) \in X^{0}$ lies in the stable set of $M_{1}=\left(u_{1}^{*}, 0\right)$, so that the unique solution $\left(u_{1}(x, t), u_{2}(x, t)\right)$ of $(5.1)$ with these initial conditions satisfies $\lim _{t \rightarrow \infty} u_{1}(\cdot, t)=u_{1}^{*}(\cdot)$ and $\lim _{t \rightarrow \infty} u_{2}(\cdot, t)=0$ uniformly in $[0,1]$. Then for any given $\epsilon>0$ there exists a $t_{0}>0$ such that for $t \geq t_{0}$,

$$
g_{2}(I(x, t))>g_{2}\left(e^{-k_{0} x-k_{1} \int_{0}^{x} u_{1}^{*}(s) d s}\right)-\epsilon, x \in[0,1] .
$$

Hence for $t \geq t_{0}, u_{2}(x, t)$ satisfies

$$
\begin{aligned}
\left(u_{2}\right)_{t} & =D_{2}\left(u_{2}\right)_{x x}+\left[g_{2}(I(x, t))-d_{2}\right] u_{2} \\
& \geq D_{2}\left(u_{2}\right)_{x x}+\left[g_{2}\left(e^{-k_{0} x-k_{1} \int_{0}^{x} u_{1}^{*}(s) d s}\right)-\epsilon-d_{2}\right] u_{2} .
\end{aligned}
$$

Comparing $u_{2}(x, t)$ with the unique solution of

$$
\begin{aligned}
& \left(U_{2}\right)_{t}=D_{2}\left(U_{2}\right)_{x x}+\left[g_{2}\left(e^{-k_{0} x-k_{1} \int_{0}^{x} u_{1}^{*}(s) d s}\right)-\epsilon-d_{2}\right] U_{2}, \\
& \left(U_{2}\right)_{x}(0, t)=0,\left(U_{2}\right)_{x}(1, t)=0, \\
& U_{2}\left(x, t_{0}\right)=\frac{1}{2} u_{2}\left(x, t_{0}\right),
\end{aligned}
$$

we deduce

$$
u_{2}(x, t)>U_{2}(x, t) \text { for } t>t_{0} .
$$

On the other hand, if $\varepsilon>0$ has been chosen sufficiently small so that $d_{2}+\varepsilon<\tilde{d}_{2}=$ $-\mu_{1}\left(-g_{2}\left(\sigma_{1}\right)\right)$, then $U_{2}(x, t) \rightarrow \infty$ as $t \rightarrow \infty$ uniformly for $x \in[0,1]$. Thus we obtain a contradiction.

Similarly, using $0<d_{1}<\tilde{d}_{1}$ we can show that $W^{s}\left(M_{2}\right) \cap X^{0}=\emptyset$.

Finally if $\left(u_{1}^{0}, u_{2}^{0}\right) \in X^{0}$ lies in the stable set of $M_{0}=(0,0)$, then we can similarly deduce that

$$
u_{2}(x, t)>U_{0}(x, t) \text { for all large } t,
$$


where $U_{0}$ is the solution of the equation for $U_{2}$ except that $u_{1}^{*}$ is replaced by 0 . Since $U_{0}(x, t) \rightarrow \infty$ we obtain a contradiction.

Thus Theorem 4.1 of [11] can be applied, and the proof is complete.

Remark 5.2. From Theorem 1.3.7 in [27], uniform persistence implies the existence of a positive steady state of (5.1). Since the assumptions in Theorem $5.1 \mathrm{imply}$ $\hat{d}_{2}<d_{2}<\tilde{d}_{2}$, this conclusion also follows from Theorem 4.3 of the previous section. On the other hand, when $d_{1}>\tilde{d}_{1}$ and $d_{2}>\tilde{d}_{2}$, we have $\hat{d}_{2}>d_{2}>\tilde{d}_{2}$, and hence (5.1) has a positive steady state by Theorem 4.3. However, it is easily checked that in this case (5.1) is not uniformly persistent.

THEOREM 5.3 (extinction). Let $\left(u_{1}, u_{2}\right)$ be the unique solution of (5.1) with initial data $u_{i}^{0} \in \dot{P}(i=1,2)$. Then the following conclusions hold:

(1) If $d_{1} \geq d_{1}^{*}$ and $d_{2} \geq d_{2}^{*}$, then $\lim _{t \rightarrow \infty} u_{1}(x, t)=0$ and $\lim _{t \rightarrow \infty} u_{2}(x, t)=0$ uniformly in $x \in[0,1]$.

(2) If $0<d_{1}<d_{1}^{*}$ and $d_{2} \geq d_{2}^{*}$, then $\lim _{t \rightarrow \infty} u_{1}(x, t)=u_{1}^{*}(x)$ and $\lim _{t \rightarrow \infty} u_{2}(x, t)$ $=0$ uniformly in $x \in[0,1]$.

(3) If $d_{1} \geq d_{1}^{*}$ and $0<d_{2}<d_{2}^{*}$, then $\lim _{t \rightarrow \infty} u_{1}(x, t)=0$ and $\lim _{t \rightarrow \infty} u_{2}(x, t)=$ $u_{2}^{*}(x)$ uniformly in $x \in[0,1]$.

Proof. Suppose that $d_{1} \geq d_{1}^{*}$ and $d_{2} \geq d_{2}^{*}$. For $i=1,2$, from the equation for $u_{i}$ we obtain

$$
\left(u_{i}\right)_{t}-D_{i}\left(u_{i}\right)_{x x} \leq\left[g_{i}\left(e^{-k_{0} x-\int_{0}^{x} k_{i} u_{i} d y}\right)-d_{i}\right] u_{i} .
$$

Applying the strong maximum principle to the equation satisfied by $u_{i}$ we deduce $u_{i}(x, 1)>0$ in $[0,1]$. Let $u_{i}^{0}=\frac{1}{2} u_{i}(x, 1)$ and let $U_{i}$ be the unique solution to

$$
\begin{cases}U_{t}=D_{i} U_{x x}+\left[g_{i}\left(e^{-k_{0} x-\int_{0}^{x} k_{i} U d y}\right)-d_{i}\right] U, & 0<x<1, t>0, \\ U_{x}(0, t)=U_{x}(1, t)=0, & t>0, \\ U(x, 0)=u_{i}^{0}(x), & 0<x<1 .\end{cases}
$$

By Lemma 3.1 we deduce that $v_{i}(x, t+1)<V_{i}(x, t)$ for $t>0$ and $x \in(0,1]$, where $v_{i}(x, t)=\int_{0}^{x} u_{i}(y, t) d y, V_{i}(x, t)=\int_{0}^{x} U_{i}(y, t) d y$.

Since $d_{i} \geq d_{i}^{*}$, from our results in section 3 we know that $\lim _{t \rightarrow \infty} U_{i}(x, t)=0$ uniformly in $x \in[0,1]$. It follows that $\lim _{t \rightarrow \infty} V_{i}(x, t)=0$ uniformly in $x \in[0,1]$ and hence $\lim _{t \rightarrow \infty} v_{i}(x, t)=0$ uniformly in $x \in[0,1]$. We may now use (5.4) and parabolic regularity much as in the proof of Theorem 3.3 to conclude that $\lim _{t \rightarrow \infty} u_{i}(x, t)=0$ uniformly in $x \in[0,1]$. This proves conclusion (1).

We next prove conclusion (2). Since $d_{2} \geq d_{2}^{*}$, the above argument can be repeated to show that $\lim _{t \rightarrow \infty} u_{2}(x, t)=0$ uniformly in $x \in[0,1]$. Therefore for any given small $\epsilon>0$ we can find $t_{0}>0$ such that

$$
g_{1}(I(x, t)) \in\left[g_{1}\left(e^{-k_{0} x-\int_{0}^{x} k_{1} u_{1} d y}\right)-\epsilon, g_{1}\left(e^{-k_{0} x-\int_{0}^{x} k_{1} u_{1} d y}\right)+\epsilon\right]
$$

for all $t \geq t_{0}$ and $x \in[0,1]$. It follows that for such $x$ and $t$,

$$
\begin{aligned}
& \left(u_{1}\right)_{t}-D_{1}\left(u_{1}\right)_{x x} \geq\left[g_{1}\left(e^{-k_{0} x-\int_{0}^{x} k_{1} u_{1} d y}\right)-\epsilon-d_{1}\right] u_{1}, \\
& \left(u_{1}\right)_{t}-D_{1}\left(u_{1}\right)_{x x} \leq\left[g_{1}\left(e^{-k_{0} x-\int_{0}^{x} k_{1} u_{1} d y}\right)+\epsilon-d_{1}\right] u_{1} .
\end{aligned}
$$

Let $u_{\epsilon}(x, t)$ be the unique solution to

$$
\begin{cases}u_{t}=D_{1} u_{x x}+\left[g_{1}\left(e^{-k_{0} x-\int_{0}^{x} k_{i} u d y}\right)-\epsilon-d_{1}\right] u, & 0<x<1, t>t_{0}, \\ u_{x}(0, t)=u_{x}(1, t)=0, & t>t_{0}, \\ u\left(x, t_{0}\right)=\frac{1}{2} u_{1}\left(x, t_{0}\right), & 0<x<1 .\end{cases}
$$

Copyright ( $)$ by SIAM. Unauthorized reproduction of this article is prohibited. 
Then by Theorem 3.3 we have

$$
\lim _{t \rightarrow \infty} u_{\epsilon}(x, t)=u_{d_{1}+\epsilon}^{*}(x)
$$

uniformly in $x \in[0,1]$, where $u_{d_{1}+\epsilon}^{*}$ denotes the unique positive steady state of the above problem.

By Lemma 3.1 we deduce

$$
v_{1}(x, t):=\int_{0}^{x} u_{1}(y, t) d y>\int_{0}^{x} u_{\epsilon}(y, t) d y \text { for } t>t_{0}, x>0 .
$$

It follows that

$$
\liminf _{t \rightarrow \infty} v_{1}(x, t) \geq \int_{0}^{x} u_{d_{1}+\epsilon}^{*}(y) d y .
$$

Letting $\epsilon \rightarrow 0$, we obtain

$$
\liminf _{t \rightarrow \infty} v_{1}(x, t) \geq \int_{0}^{x} u_{d_{1}}^{*}(y) d y .
$$

Similarly, if $u^{\epsilon}$ is the unique solution to

$$
\begin{cases}u_{t}=D_{1} u_{x x}+\left[g_{1}\left(e^{-k_{0} x-\int_{0}^{x} k_{i} u d y}\right)+\epsilon-d_{1}\right] u, & 0<x<1, t>t_{0}, \\ u_{x}(0, t)=u_{x}(1, t)=0, & t>t_{0}, \\ u\left(x, t_{0}\right)=\frac{3}{2} u_{1}\left(x, t_{0}\right), & 0<x<1,\end{cases}
$$

then we can make use of Theorem 3.3 and Lemma 3.1 to deduce that

$$
\limsup _{t \rightarrow \infty} v_{1}(x, t) \leq \int_{0}^{x} u_{d_{1}-\epsilon}^{*}(y) d y
$$

Letting $\epsilon \rightarrow 0$, we obtain

$$
\limsup _{t \rightarrow \infty} v_{1}(x, t) \leq \int_{0}^{x} u_{d_{1}}^{*}(y) d y .
$$

Thus we must have

$$
\lim _{t \rightarrow \infty} v_{1}(x, t)=\int_{0}^{x} u_{d_{1}}^{*}(y) d y .
$$

We may now use (5.4) and the parabolic regularity theory to conclude, as in the proof of Theorem 3.3, that $u_{1}(x, t) \rightarrow u_{d_{1}}^{*}(x)$ as $t \rightarrow \infty$ uniformly in $x \in[0,1]$. This proves conclusion (2).

The proof of conclusion (3) is parallel to the proof of (2) above.

Theorem 5.1 can be extended to the $n \geq 3$ species case. A complete understanding of the dynamical behavior for the model with three or more species seems out of reach at the moment. For the two-species model, we conjecture the following:

1. If $0<d_{2}<\tilde{d}_{2}$ and $\tilde{d}_{1}<d_{1}<d_{1}^{*}$, then $\lim _{t \rightarrow \infty} u_{1}(x, t)=0$ and $\lim _{t \rightarrow \infty} u_{2}$ $(x, t)=u_{2}^{*}(x)$ uniformly in $x \in[0,1]$.

2. If $0<d_{1}<\tilde{d}_{1}$ and $\tilde{d}_{2}<d_{2}<d_{2}^{*}$, then $\lim _{t \rightarrow \infty} u_{1}(x, t)=u_{1}^{*}(x)$ and $\lim _{t \rightarrow \infty} u_{2}(x, t)=0$ uniformly in $x \in[0,1]$.

3. If $\tilde{d}_{1}<d_{1}<d_{1}^{*}$ and $\tilde{d}_{2}<d_{2}<d_{2}^{*}$, then the competition outcomes depend on the initial data.

4. If $0<d_{1}<\tilde{d}_{1}$ and $0<d_{2}<\tilde{d}_{2}$, then there exists a unique positive steady state $\left(u_{1}^{c}(\cdot), u_{2}^{c}(\cdot)\right)$ such that $\lim _{t \rightarrow \infty} u_{1}(x, t)=u_{1}^{c}(x), \lim _{t \rightarrow \infty} u_{2}(x, t)=u_{2}^{c}(x)$ uniformly in $x \in[0,1]$ for any initial data $u_{i}^{0} \in \dot{P}(i=1,2)$.

Copyright $@$ by SIAM. Unauthorized reproduction of this article is prohibited. 
Acknowledgment. Y. Du would like to thank Professor Yaping Wu (Capital Normal University, Beijing) for some early discussions on the convergence problem of the single-species equation.

\section{REFERENCES}

[1] R. A. Armstrong And R. McGehee, Competitive exclusion, Amer. Naturalist, 115 (1980), pp. 151-170.

[2] J. BLAT AND K. J. Brown, Global bifurcation of positive solutions in some systems of elliptic equations, SIAM J. Math. Anal., 17 (1986), pp. 1339-1353.

[3] M. G. Crandall and P. H. Rabinowitz, Bifurcation from simple eigenvalues, J. Funct. Anal., 8 (1971), pp. 321-340.

[4] E. N. DANCER, AND Y. Du, Positive solutions for a three-species competition system with diffusion. I. General existence results, Nonlinear Anal., 24 (1995), pp. 337-357.

[5] Y. Du, Bifurcation from semitrivial solution bundles and applications to certain equation systems, Nonlinear Anal., 27 (1996), pp. 1407-1435.

[6] Y. Du, A degree theoretic approach to $N$-species periodic competition systems on the whole $R^{n}$, Proc. Roy. Soc. Edinburgh Sect. A, 129 (1999), pp. 295-318.

[7] Y. Du AND S.-B. Hsu, Concentration phenomena in a nonlocal quasi-linear problem modelling phytoplankton I: Existence, SIAM J. Math. Anal., 40 (2008), pp. 1419-1440.

[8] Y. DU, AND S.-B. Hsu, Concentration phenomena in a nonlocal quasi-linear problem modelling phytoplankton II: Limiting profile, SIAM J. Math. Anal., 40 (2008), pp. 1441-1470.

[9] U. Ebert, M Arrayas, N. Temme, B. Sommeijer, and J. Huisman, Critical condition for phytoplankton blooms, Bull. Math. Biol., 63 (2001), pp. 1095-1124.

[10] K. Fennel And E. Boss, Subsurface maxima of phytoplankton and chlorophyll: Steady-state solutions from a simple model, Limnol. Oceanogr., 48 (2003), pp. 1521-1534.

[11] J. K. Hale and P. Waltman, Persistence in infinite-dimensional systems, SIAM J. Math. Anal., 20 (1989), pp. 388-395.

[12] S. B. Hsu, Limiting behavior for competing species, SIAM J. Appl. Math., 34 (1978), pp. 760-763.

[13] S. B. Hsu, S. Hubbell, and P. Waltman, A mathematical theory for single-nutrient competition in continuous cultures of micro-organisms, SIAM J. Appl. Math., 32 (1977), pp. 366-383.

[14] J. Huisman, M. Arrayas, U. Ebert, and B. Sommeijer, How do sinking phytoplankton species manage to persist?, Amer. Naturalist, 159 (2002), pp. 245-254.

[15] J. Huisman, N. N. Pham Thi, D. M. Karl, and B. Sommeijer, Reduced mixing generates oscillations and chaos in the oceanic deep chlorophyll maximum, Nature, 439 (2006), pp. $322-325$.

[16] J. Huisman, P. van Oostveen, and F. J. Weissing, Species dynamics in phytoplankton blooms: Incomplete mixing and competition for light, Amer. Naturalist, 154 (1999), pp. $46-67$.

[17] J. Huisman And F. J. Weissing, Light-limited growth and competition for light in well-mixed aquatic environments: An elementary model, Ecology, 75 (1994), pp. 507-520.

[18] J. Huisman And F. J. Weissing, Competition for nutrients and light in a mixed water column: A theoretical analysis, Amer. Naturalist, 146 (1995), pp. 536-564.

[19] H. IsHII AND I. TAKAGI, Global stability of stationary solutions to a nonlinear diffusion equation in phytoplankton dynamics, J. Math. Biol., 115 (1982), pp. 65-92.

[20] C. A. Klausmeier and E. E. Litchman, Algal games: The vertical distribution of phytoplankton in poorly mixed water columns, Limnol. Oceanogr., 46 (2001), pp. 1998-2007.

[21] T. Kolokolnikov, C. Ou, And Y. Yuan, Phytoplankton depth profiles and their transitions near the critical sinking velocity, J. Math. Biol., 59 (2009), pp. 105-122.

[22] P. H. Rabinowitz, Some global results for nonlinear eigenvalue problems, J. Funct. Anal., 7 (1971), pp. 487-513.

[23] D. Tilman, Resource Competition and Community Structure, Princeton University Press, Princeton, NJ, 1982.

[24] S. Tотаво, Mutual shading effect on algal distribution: A nonlinear problem, Nonlinear Anal., 13 (1989), pp. 969-986.

[25] F. J. Weissing and J. Huisman, Growth and competition in a light gradient, J. Theoret. Biol., 168 (1994), pp. 323-336.

[26] A. Zagaris, A. Doelman, N. N. Pham Thi, and B. P. Sommeijer, Blooming in a nonlocal, coupled phytoplankton-nutrient model, SIAM J. Appl. Math., 69 (2009), pp. 1174-1204.

[27] X. Q. Zhaо, Dynamical Systems in Population Biology, Springer-Verlag, New York, 2003.

Copyright (c) by SIAM. Unauthorized reproduction of this article is prohibited. 